Bond University

Research Repository

\title{
Extrahippocampal contributions to spatial navigation in humans: A review of the neuroimaging evidence
}

Baumann, Oliver; Mattingley, Jason B.

Published in:

Hippocampus

DOI:

10.1002/hipo. 23313

Licence:

Other

Link to output in Bond University research repository.

Recommended citation(APA):

Baumann, O., \& Mattingley, J. B. (2021). Extrahippocampal contributions to spatial navigation in humans: A review of the neuroimaging evidence. Hippocampus, 31(7), 640-657. https://doi.org/10.1002/hipo.23313

\section{General rights}

Copyright and moral rights for the publications made accessible in the public portal are retained by the authors and/or other copyright owners and it is a condition of accessing publications that users recognise and abide by the legal requirements associated with these rights.

For more information, or if you believe that this document breaches copyright, please contact the Bond University research repository coordinator. 
Extrahippocampal contributions to spatial navigation in humans: a review of the neuroimaging evidence

\section{Oliver Baumann ${ }^{1 *}$ \& Jason B. Mattingley ${ }^{2,3,4}$}

${ }^{1}$ School of Psychology, Bond University, Robina, QLD 4226

${ }^{2}$ Queensland Brain Institute, The University of Queensland, Brisbane, QLD 4072 Australia

${ }^{3}$ School of Psychology, The University of Queensland, Brisbane, QLD 4072 Australia

${ }^{4}$ Canadian Institute for Advanced Research (CIFAR)

*obaumann@bond.edu.au 


\begin{abstract}
Spatial navigation is a crucial everyday skill, which when impaired leads to a significant decrease in quality of life. In humans, functional magnetic resonance imaging (fMRI) has provided extensive insights into the neural underpinnings of navigation skills. Whereas the hippocampus has been recognized as the prime region underpinning navigation abilities, by providing a cognitive map of the environment, imaging studies have also implicated a range of other brain regions. In this review we provide an overview of the fMRI evidence for extrahippocampal contributions to spatial navigation. We show that the parahippocampal cortex, retrosplenial cortex, dorsal striatum and the posterior parietal cortex provide important complementary functions, and ultimately form part of a functional network that regulates successful wayfinding behaviour.
\end{abstract}




\section{Extrahippocampal contributions to spatial navigation in humans: a review of the}

\section{neuroimaging evidence}

Spatial navigation is the act of traversing the environment in a purposeful manner. At the core of spatial navigation lies the ability to analyse, encode and retrieve relevant characteristics of the environment, and to use this information to manoeuvre the body to reach a desired spatial location. Relevant environmental characteristics range from simple, well-defined entities such as objects, landmarks and scenes, to more complex and abstract concepts such as directions, routes and geometric layouts. The complexity of these environmental characteristics is reflected in the neuro-cognitive mechanisms that underpin their processing. In mammals, including humans, the hippocampus is arguably the prime structure for forming representations of the spatial environment. The earliest reports of a role for the hippocampus in spatial encoding began to emerge in the 1960s and 70s (Stein \& Kimble, 1966; Means \& Douglas, 1970). Work at that time was based principally on lesion studies and electrophysiological recordings in rats and monkeys. One of the most prominent and widely cited examples is John O'Keefe's discovery of dedicated cells in the rat hippocampus that encode allocentric spatial information - so-called place cells (O’Keefe \& Dostrovsky, 1971). While this discovery was received with interest at the time, the number of studies investigating hippocampal contributions to spatial encoding grew exponentially over subsequent decades (from 66 publications in the 1970s to 360 in the 1980s, culminating in well over 8,000 published works in the last 10 years).

The widespread use of behavioural paradigms such as the Morris Water Maze to explore memory processes in rodents (Morris, 2008) was based at least in part on the observation that rats have excellent spatial abilities, as well as the fact that spatial performance can be 
precisely quantified and replicated using such tasks. Studies of hippocampal function based on animal models have provided detailed causal insights at the level of individual neurons and local neuronal circuits. The scientific impact of this work culminated in 2014 with the award of the Nobel Prize in Physiology or Medicine to John O'Keefe, May-Britt Moser, and Edvard Moser, for their work in rodents on hippocampal and entorhinal mechanisms underlying mental representations of space (Burgess, 2014). Critically, however, humans differ from rodents not only in terms of their perceptual, cognitive and motor abilities, but also in terms of brain architecture and functional organization. It thus remains unclear to what extent it is possible to extrapolate findings from animal models, including rodents and monkeys, to humans (Clark \& Squire, 2013).

Early human lesion studies on the functional properties of the hippocampus focused predominantly on verbal paradigms and the examination of episodic and semantic memory processes (e.g. Scoville \& Milner, 1957; Milner et al. 1968). Later human lesion work, however, also uncovered an important role of the hippocampus in spatial memory and wayfinding behaviour. For instance, Abrahams and colleagues (1997) assessed memory deficits in epileptic patients with right hippocampal damage using a task that required the patients either to encode the positions of identical containers, or the identity of objects placed within the containers. Abrahams et al. (1997) observed a selective impairment for spatial learning of position information in their right hippocampal patients, whereas object learning remained relatively intact. These and similar results (e.g. Bohbot et al. 1998) support the idea of a hippocampal role in spatial processing, as suggested by the earlier animal studies.

While human lesion studies potentially allow causal inferences regarding brain-behaviour relationships, as well as a high degree of ecological validity, they come with two principal 
disadvantages. First, human lesions by their nature are typically not well circumscribed, given that they arise from pathophysiological conditions that do not respect functional anatomical boundaries, such as stroke, tumour or anoxic damage, and that they often lead to diverse cognitive deficits. This in turn leads to considerable variability in perceptual and cognitive profiles between individuals, which can complicate attempts to relate brain structure to function. Second, lesion studies are typically focussed on single brain regions and therefore do not account for functional impairments that might arise in distant but functionally connected areas. These disadvantages can be overcome by employing functional neuroimaging in healthy human volunteers. In this paper, we focus on discoveries arising from the most popular of these methods, functional magnetic resonance imaging (fMRI), which allows researchers to measure whole brain activity in vivo with a high degree of spatial precision ( $\sim$ up to $1 \mathrm{~mm}$ in $\mathrm{fMRI}$; e.g. Suthana et al. 2015) and reasonable temporal resolution (up to $10 \mathrm{~Hz}$; LeVan et al. 2018).

As with any measurement technique in science, and despite its unique advantages for studying spatial navigation, functional neuroimaging also has its weaknesses. Arguably the biggest disadvantage is that neuroimaging requires immobilisation of the participant's head during image acquisition. This makes it impossible to record brain signals during actual navigation, unlike rodent electrophysiological studies that can employ wireless recording techniques (e.g. Fan et al. 2011). One strategy to compensate for this limitation is the use of "virtual navigation" paradigms, a strategy employed in several of the studies we consider in this review. In such paradigms, participants use MRI-compatible response devices to navigate their way through computer-generated 2-D or 3-D virtual environments. 
While the first neuroimaging investigations of spatial navigation in humans focussed on the hippocampus as a key region of interest (Maguire et al., 1997; O'Keefe et al., 1998), based mainly on evidence from early animal studies, this work also revealed activations in a range of other brain regions, including the medial temporal cortex and subregions of the parietal and frontal cortices (Aguirre et al., 1996; Maguire et al., 1996,1997; Ghaem et al., 1997). It is now clear that in humans, processes related to spatial navigation and the mental representation of space rely on a widespread network of areas beyond the hippocampus (e.g., Baumann et al. 2010, 2012). Here we provide an overview of recent research on the role played by some of these extra-hippocampal regions in spatial cognition. We focus in particular on the parahippocampal cortex, the retrosplenial complex, the dorsal striatum, and the posterior parietal cortex (see Fig. 1), as these four regions are most consistently implicated, together with the hippocampus, in spatial cognition and wayfinding.

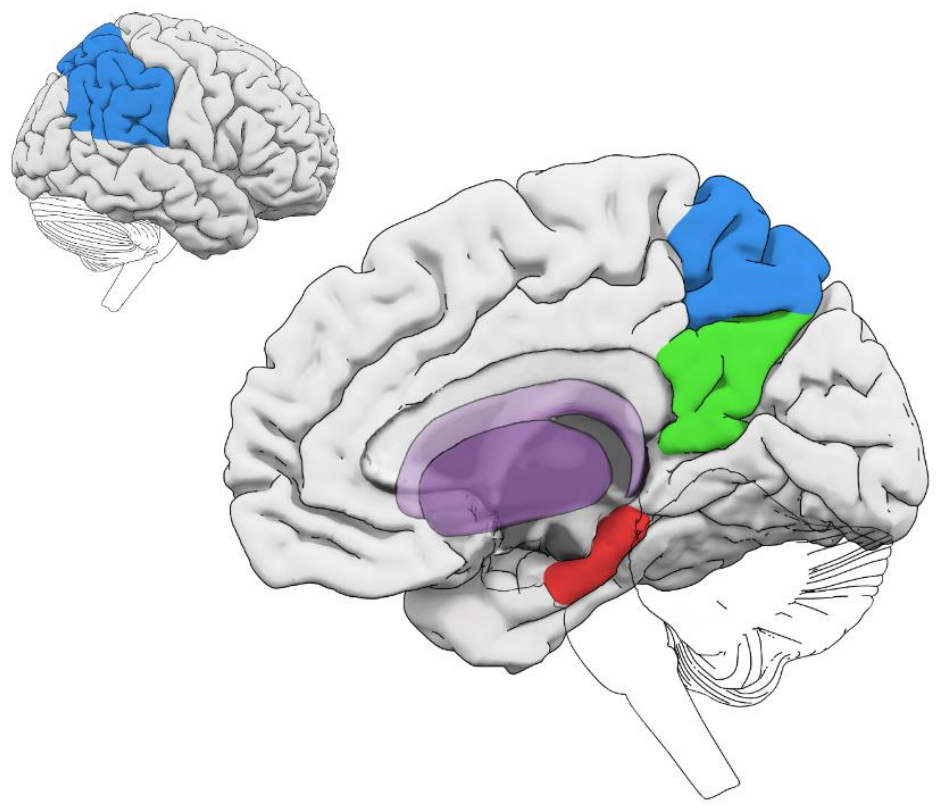

Figure 1. Schematics of the brain regions underlying spatial navigation. Red=Parahippocampal gyrus; Green $=$ Retrosplenial complex; Purple = Dorsal striatum; Blue $=$ Posterior parietal cortex). 


\section{Parahippocampal Cortex}

In humans, the parahippocampal cortex is typically defined as the posterior section of parahippocampal gyrus, i.e. the neo-cortical region posterior to the entorhinal cortex and perirhinal cortex (see Fig. 1). Early evidence for the involvement of the parahippocampal cortex in spatial navigation was provided by Aguirre and colleagues (1996), who employed a modified computer game to create a virtual reality maze that was presented to participants in first-person view. The maze was simple, containing just three, 3-way intersections and five cul-de-sacs. During learning epochs, participants were invited to freely explore the maze using a four-button, fibre-optic control pad, and to memorize the locations of four landmarks. These learning epochs alternated with control epochs, in which participants navigated an endlessly looping corridor. Aguirre and colleagues found that parahippocampal activity was significantly higher in the active learning condition than in the control condition, which also involved spatial locomotion within the virtual environment, but which presumably did not engage navigational processes or spatial memory systems. While the task design and MRI hardware employed by Aguirre and colleagues were fairly rudimentary by current standards in cognitive neuroscience, particularly in terms of both the temporal and spatial resolution of the images obtained, their work nevertheless provided an early clue to the involvement of the parahippocampal cortex in spatial navigation.

The essential role of the parahippocampal gyrus in spatial navigation was further demonstrated in a later experiment (Baumann et al. 2010), which aimed to establish whether this region is important for navigational behaviour, beyond a more general involvement (e.g., due to higher task demands in active versus passive scenarios). The study used fMRI to investigate whether there is a predictive relationship between navigation-related brain 
activity and spatial accuracy in a virtual navigation task. Participants manoeuvred through a sparse environment, which consisted of an infinite floor populated with four geometrical shapes, which served as landmarks. Inclusion of a single target object allowed for effective control of the relative salience of paths and landmarks, and thus served to limit variability in behavioural strategies. On each trial, participants were initially required to navigate to the target object using a joystick. Following a short delay period, they were instantaneously transported to a new location and asked to navigate back to the remembered location of the target object, which had now been removed from the environment. A regression analysis indicated that the magnitude of parahippocampal activity during initial encoding of the target was reliably associated with subsequent navigational accuracy to its remembered location. That is, on trials in which encoding-related parahippocampal activity was high, participants located the target more accurately during the retrieval phase than when parahippocampal activity was low. This predictive relationship between parahippocampal activity and navigation performance provides further evidence for a functional role of this cortical area in spatial navigation. Critically, these findings also raise the question of the degree to which parahippocampal activity might be modulated by attentional and mnemonic affordances in navigation tasks.

This question was addressed explicitly in a subsequent study by Janzen \& van Turennout (2004), who used fMRI to investigate whether objects with navigational relevance (i.e., objects located at critical intersections or "decision points") within a virtual environment are processed differently from objects without navigational relevance (see Fig 2a). 


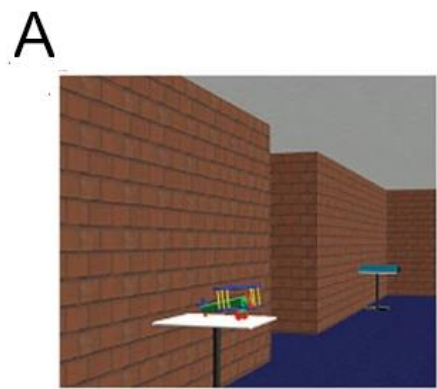

$\mathrm{B}$

Toy at decision point

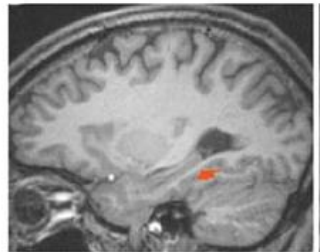

$x=26$

C

Right parahippocampal gyrus

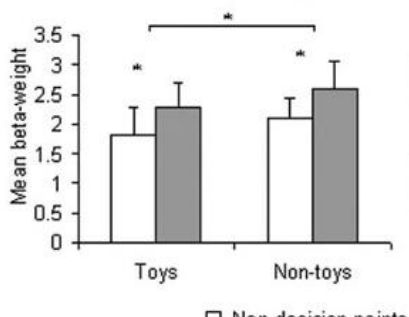

$\square$ Non-decision points

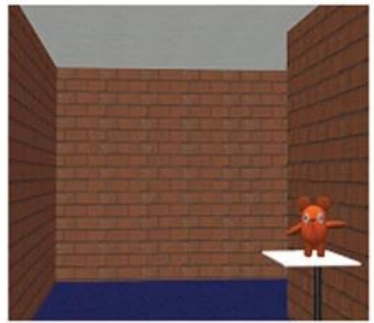

Toy at non-decision point

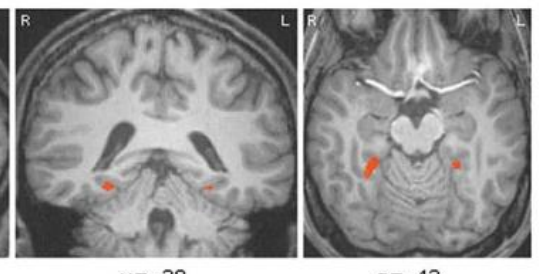

$z=-12$

$y=-39$

Left parahippocampal gyrus

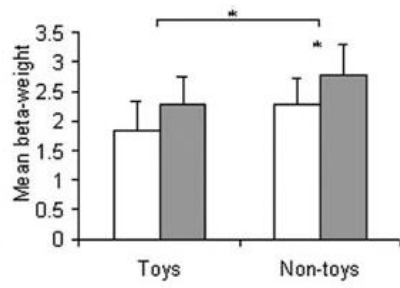

$\square$ Decision points

Figure 2. Static views of example decision points in the virtual environment employed by Janzen \& van Turennout (2004), and associated fMRI activity in the parahippocampal cortex.

(A) First-person view of the virtual environment, showing the placement of objects (toys) at decision points (i.e., intersections) and non-decision points (i.e., corners). (B) Brain images showing higher activity in the left and right parahippocampal cortex during the recognition phase, for trials in which participants were shown images of objects located at decision-points compared with non-decision points. (C) Mean beta weights of the activation maxima in the left and right parahippocampal gyrus, showing that activity in response to decision-point objects was higher than activity to non-decision point objects, even if their learning was not required per task instruction (i.e., non-toys). Figure adapted with permission from Janzen \& van Turennout (2004). 
First, participants were asked to navigate and learn the layout of a virtual "museum" before entering the scanner. Importantly, participants' attention to the environment was controlled by having them focus only on locations at which objects of a specified category (toys) appeared. In a subsequent recognition phase, which was conducted within the scanner, participants were shown snapshot images from the previously learned environment, intermingled with novel lures, and were asked to indicate which objects they recognized. Importantly, participants were not informed that navigational relevance was of importance for the experimental hypothesis. Comparing the activation profile of navigationally-relevant objects and navigationally-irrelevant objects, the authors found that parahippocampal activity was significantly higher for navigationally-relevant objects (see Fig 2b). Strikingly, this pattern held even for objects that participants failed to remember, implying that parahippocampal representations of navigationally relevant objects does not require conscious awareness (see Fig 2c).

A later study by Chan and colleagues (2014) used fMRI to investigate whether parahippocampal representations of spatial location can be modulated by factors other than navigational relevance. More specifically, the authors asked whether the emotional state of the navigator, induced by task-irrelevant, affective visual stimuli, can influence spatial representations and their associated activity patterns in navigationally relevant areas of a learned environment. In the first phase of the study, participants actively navigated through a virtual apartment over several sessions, with the goal of learning its spatial layout in terms of the rooms it contained and the objects within them. 
After each object-location learning trial, participants were shown an image from the International Affective Picture System (IAPS; Lang, Bradley, \& Cuthbert, 1999) with the aim of eliciting either a positive, negative, or neutral emotion. Using this procedure, each room of the virtual apartment was effectively associated with a different emotional valence, even though participants were not aware of the association based on their responses to a poststudy survey. In a second stage of the experiment conducted a day after the learning phase, participants' fMRI brain activity was recorded while their knowledge of the object-room associations was probed. Importantly, this phase of the study used only static images of the rooms and objects; no emotional images were shown. The authors found that activity in the bilateral parahippocampal cortex was significantly higher when participants viewed snapshots of rooms that had been (unconsciously) associated with negative stimuli during the learning phase, relative to snapshots of rooms associated with neutral or positive stimuli. This difference in brain activity was also accompanied by significantly faster reaction times for the rooms that had been associated with negative stimuli. Interestingly, when queried participants denied being aware of the emotional valence associated with each room. Moreover, the modulatory effect was only present in the parahippocampal cortex, and not in other navigationally relevant areas, including the hippocampus. Taken together with the findings of Janzen \& van Turennout (2004), the work of Chan et al. (2014) not only suggests that the parahippocampal cortex plays an important role in implicit learning of spatial information, but also that the encoding processes it supports are sensitive to emotionallyrelevant stimuli, ultimately aiding efficient and responsive navigational behaviour.

In recent years two dominant hypotheses have emerged for the functional role of the parahippocampal cortex in spatial navigation. One hypothesis proposes that the parahippocampal cortex is involved in the perceptual analysis of scene layouts (Epstein et al., 
1999), whereas the other suggests that it is primarily involved in encoding and retrieval of associative contextual memories (Aminoff et al. 2013). In a series of experiments aimed at addressing these hypotheses, Epstein and colleagues showed that the parahippocampal cortex responds strongly to visual scenes such as landscapes, cityscapes and buildings (e.g. Epstein and Kanwisher, 1998; Epstein, 2008; Epstein and Ward, 2010). For instance, Epstein and colleagues (1999) recorded fMRI activity from participants while they viewed images of faces, objects, empty rooms or rooms containing objects. The latter three classes of stimuli were either photographs of real exemplars, or else were depictions of matching items constructed from Lego ${ }^{\mathrm{TM}}$. Whereas faces did not elicit any signal change relative to baseline in the parahippocampal cortex, there was moderate activity in this region for objects (real and Lego), higher activity for rooms made of Lego, and highest activity for images of real rooms. Most notably, Lego rooms elicited higher activity than Lego objects, even though they were both made from the same material and the Lego room was quite obviously not a real "place". Moreover, for the images of real rooms there was no activity difference for those that were empty versus furnished.

The spatial layout hypothesis received further support from a study by Park et al. (2011), which used fMRI to measure neural responses to real-world scenes that varied systematically in their spatial layout (closed vs. open), and content (natural vs. open), as shown in Figure 3. The experiment required participants to attend to a series of images while performing an incidental 1-back task to maintain alertness. A multivoxel pattern analysis was used to determine whether responses in five regions of interest could be classified based on the image categories of 'boundary' (i.e. spatial layout) and 'content' (i.e. texture). Activity patterns in the parahippocampal cortex were decodable for the dimension of boundary but 
were insensitive to the content of the scene. By contrast, activity patterns in the lateral occipital cortex were decodable for the content dimension, but not the boundary dimension.

These findings were extended in a study by Kravitz et al. (2011a), which used a similar experimental design and employed representational similarity analysis of multivoxel patterns to discriminate activity along the three chosen dimensions of real-world scene images. Two of those dimensions were comparable to those of Park and colleagues (2011), i.e. content (man-made vs. natural), and expanse (open vs. closed), but they also added a third dimension of relative distance, i.e. the distance between viewer and foreground objects (near vs. far). Consistent with Park et al. (2011), activity in the parahippocampal cortex was successfully decoded for the spatial dimensions of expanse and distance, but not for the dimension of scene content. Taken together, the studies of Park et al. (2011) and Kravitz et al. (2011a) support the suggestion that the parahippocampal cortex is concerned with the visuo-spatial analysis of scene layouts.
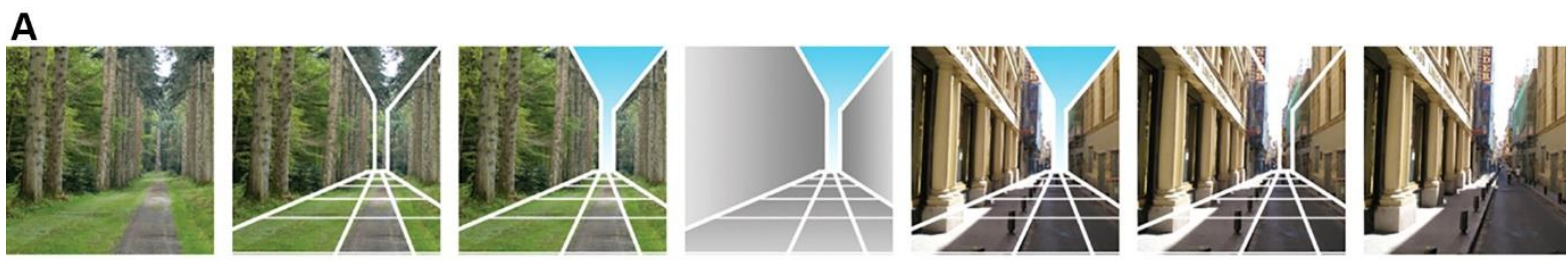

NATURAL content CLOSED spatial boundary URBAN content

B
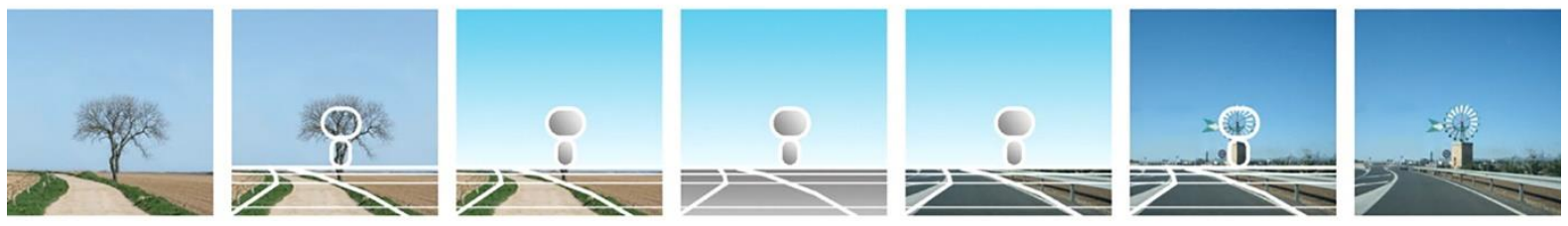

NATURAL content

OPEN spatial boundary URBAN content

Figure 3. Schematic of the visual stimuli used by Park et al. (2011) to assess neural acitvation patterns in response to variations in the dimensions of spatial boundary and scene content. (A) Closed boundary scenes with natural vs. urban content. (B) Open boundary scenes with 
natural vs. urban content. Figure by Park et al. (2011), used under a Creative Commons Attribution 4.0 International (CC BY 4.0) license.

The findings reviewed above suggest that parahippocampal activity is driven by the presence of spatial layouts rather than by the visual complexity of the stimuli they contain, or by the recognisability of the visual information therein (hence the term "parahippocampal place area"; Epstein \& Kanwisher, 1998). Another interesting observation is that parahippocampal fMRI activity is enhanced for large objects relative to small objects, which evidently makes them more 'place-like' (Mullally \& Maguire, 2011; Konkle \& Oliva, 2012; Troiani et al., 2014; Marchette, et al., 2015). Other fMRI studies have provided insights into additional facets of the functional neuroanatomical properties of the parahippocampal cortex in visuo-spatial analysis. For instance, it has been found that the size and clutter in natural scenes, i.e. the quantity of components that fill up a scene, such as objects, people, walls and so on, are related to activity patterns in the parahippocampal cortex (Park et al., 2015).

In contrast, according to the contextual hypothesis, activity in the parahippocampal cortex reflects modality-independent retrieval of contextual information from memory, such as which objects typically co-occur in a given scene and where they are likely to be located relative to one another - for example, a refrigerator and an oven are both likely to be found in the kitchen, and they both serve a function in relation to food (Aminoff et al. 2013). Bar \& Aminoff (2003) provided support for this hypothesis using a two-stage study design. In the first stage, they surveyed a group of participants to compile lists of visual objects that were classified as having either strong contextual associations (i.e. objects strongly associated with a specific context, such as a supermarket cart) or weak contextual associations (i.e. objects that occur in many different contexts, such as a camera). Importantly, the researchers took 
care to avoid confounds with other image dimensions, such as physical properties, semantic attributes, and functions. In the second phase of the experiment, images of selected stimuli were presented to a fresh group of participants in the scanner, and they were to indicate simply whether they recognised the object. The authors found that presentation of high contextual objects significantly increased activity in the parahippocampal cortex relative to low contextual objects and suggested that this result was likely due to the automatic activation of numerous related concepts in the high context condition. Importantly, according to this interpretation, it should not matter whether relationships are presented visually or verbally.

To test this idea, Baumann \& Mattingley (2016) conducted an fMRI study which sought to compare parahippocampal activation patterns in response to visuo-spatial information with those associated with contextual mnemonic processes. While in the scanner participants were presented with object-object or object-scene pairs that appeared in either printed-word format or in a combined word-picture format, as shown in Fig 4a. Participants engaged in a task that required them to judge the contextual relatedness of scene and object pairs (e.g., "bike" and "bell" are a contextually congruent word-scene pair, whereas "tractor" and "hotel" are a contextually incongruent object-scene pair (see Fig 4a). Using a factorial design, the analysis aimed to identify brain regions that responded preferentially to scenes, and those that were selectively active during the contextual judgment task regardless of whether scenes or objects were presented. The findings revealed a functional dissociation along the longitudinal axis of the parahippocampal gyrus bilaterally (see Fig 4b). Specifically, whereas the most posterior aspect of the parahippocampal gyrus was preferentially involved in the visuospatial analysis of scenes (in line with Epstein's hypothesis; Epstein et al., 1999), the more anterior portions were involved in contextual judgements irrespective of stimulus type 
(in line with Aminoff and Bar's hypothesis; Aminoff et al. 2013). Taken together, Baumann and Mattingley's (2016) findings suggest that the posterior parahippocampal cortex supports spatial navigation performance by providing critical information about visuo-spatial aspects of the environment to the hippocampus.

This interpretation is in line with findings in both rats and monkeys which suggest that the homologues of human posterior parahippocampal cortex (areas $\mathrm{TH}$ and TF) project to hippocampal areas CA1 and pre-subiculum, which contain allocentric view cells (i.e., cells that respond when a specific element of the environment falls within their receptive fields; Matsumura et al. 1999; Robertson et al., 1999). Furthermore, data from fMRI connectivity studies suggest that anterior sections of the parahippocampal cortex are more strongly connected with parietal and medial temporal areas, whereas posterior sections are more strongly connected with occipital areas (Baldassano et al., 2013). Overall, the human imaging work summarised in this section suggests that the posterior parahippocampal gyrus underpins spatial navigation by analysing visuo-spatial properties of visual scenes. It is noteworthy that both $\mathrm{fMRI}$ and single-cell recordings in macaques have provided compelling evidence for the existence of scene-selective cortical areas in the anterior parieto-occipital sulcus, as well as in area V3A and the dorsal prelunate gyrus (Nasr et al., 2011; Kornblith, et al., 2013). 


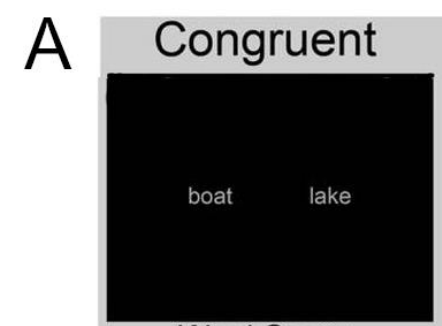

Word-Scene

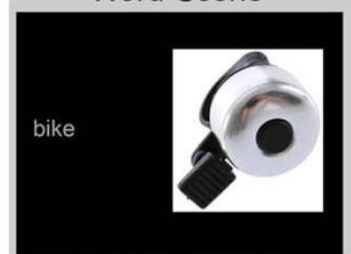

Picture-Object

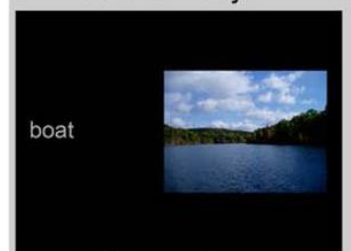

Picture-Scene

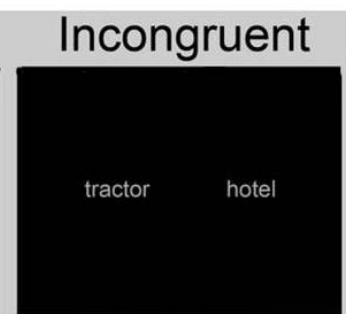

Word-Scene

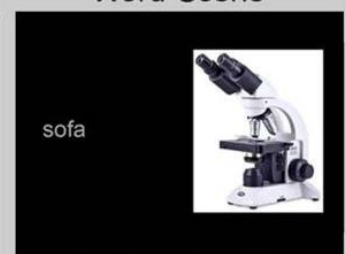

Picture-Object

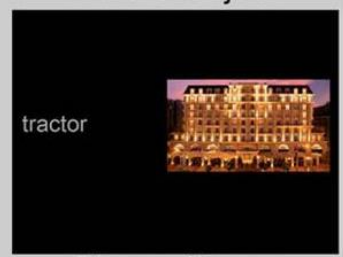

Picture-Scene

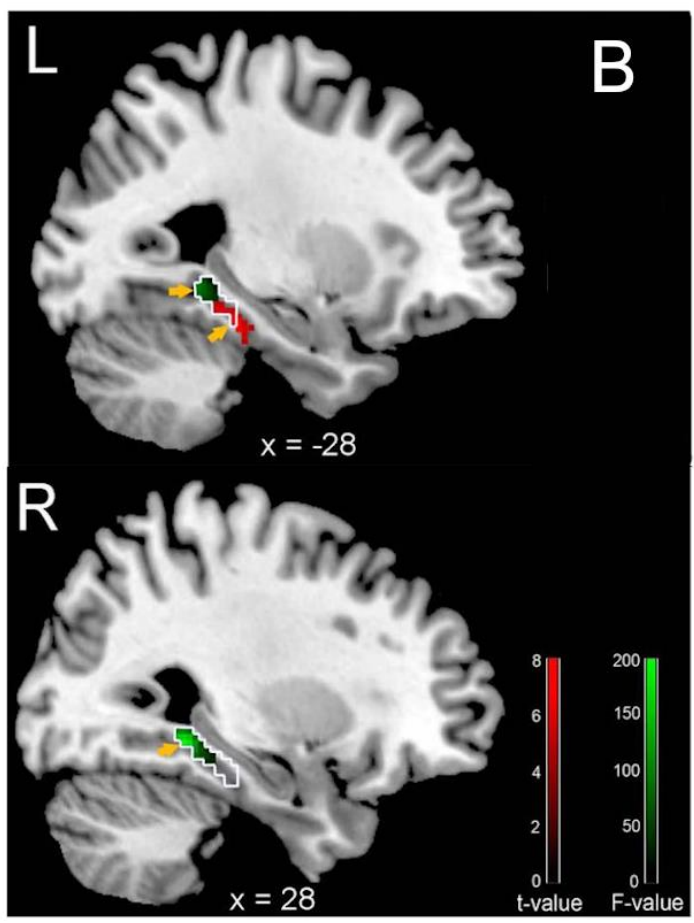

Figure 4. Schematics of the task employed by Baumann \& Mattingley (2016), and associated fMRI activation patterns. (A) Example stimuli from the different task conditions, involving pairs of objects and object-scene pairs that were either presented in word format or in a combined word-picture format. Participants were required to judge the contextual congruence of the pairs (i.e., whether co-occurrences are typical). (B) Sagittal MRI brain slices showing areas of the parahippocampal cortex involved in visuo-spatial analysis (green) and contextual retrieval (red). Yellow arrows indicate activation maxima. Figure adapted from Baumann \& Mattingley (2016) under a Creative Commons Attribution 4.0 International (CC BY 4.0) license.

\section{Retrosplenial Complex}

While the retrosplenial cortex proper encompasses Brodmann areas (BA) 29 and 30, this label is typically used more widely in human imaging studies to refer to brain areas within the 
retrosplenial/posterior cingulate/medial parietal region, which also includes areas BA23 and BA31 (see Fig 1). For this reason, Epstein (2008) suggested the term retrosplenial complex to refer to any scene-responsive regions within this broader area. With respect to its role in spatial navigation, in their classic model of spatial recall Becker and Burgess (2001) proposed that, due to its dense connectivity with parietal and medial temporal regions (Kobayashi \& Amaral, 2003), the retrosplenial cortex is ideally suited to provide an interface between two different spatial reference systems used for spatial navigation. One is the egocentric reference system, regulated by the posterior parietal cortex, which underpins navigation using a self-centered or first-person perspective to track a known path from one location to another. The second is the allocentric reference system, controlled by the medial temporal cortex, which is externally centered and can be thought of as a third-person perspective (Chrastil \& Chrastil, 2013). The idea that the retrosplenial cortex integrates across egocentric and allocentric representations is in line with the observation in rats that the retrosplenial cortex contains cells that code the animal's current heading (for an overview see Taube, 2007). Such heading-direction information is assumed to require integration of egocentric self-motion information and allocentric landmark information. Evidence from human fMRI studies has similarly demonstrated a role for the retrosplenial complex in the encoding of perceived heading (Baumann \& Mattingley, 2010; Marchette et al. 2014).

In a study by Baumann \& Mattingley (2010), participants initially completed five daily learning sessions within a virtual 10-corridor ( $5 \times 5$ ) Cartesian grid (Fig 5a). At the end of each corridor there was a unique pictorial landmark (Fig 5b). The learning task required participants to navigate between landmarks as quickly and efficiently as they could. The second phase of the experiment was conducted in the scanner and required participants to view snapshots taken from within the virtual environment and to judge the position of the vantage point relative to 
the centre of the virtual grid. This task was instrumental for engaging participants in retrieving the spatial aspects of the virtual environment, but orthogonal to the question of which brain regions were sensitive to differences in perceived heading. The data analysis, which focused on neural adaptation effects in the $\mathrm{fMRI}$ signal in response to repeated presentations of the same heading (compared with orthogonal headings), revealed that the retrosplenial complex (i.e., BA31) alone was modulated by perceived heading (see Fig 5c). More recently, it has been shown that heading sensitivity of the human retrosplenial cortex applies not only to directions in the horizontal plane but also to those in the vertical plane (i.e., relative to locations above or below the observer; Kim \& Maguire, 2019). Moreover, Vass and Epstein (2017) found that the retrosplenial complex retains its sensitivity to heading information even in tasks that require orienting oneself in imagined environments, producing highly similar results to those obtained in visual orientation tasks.
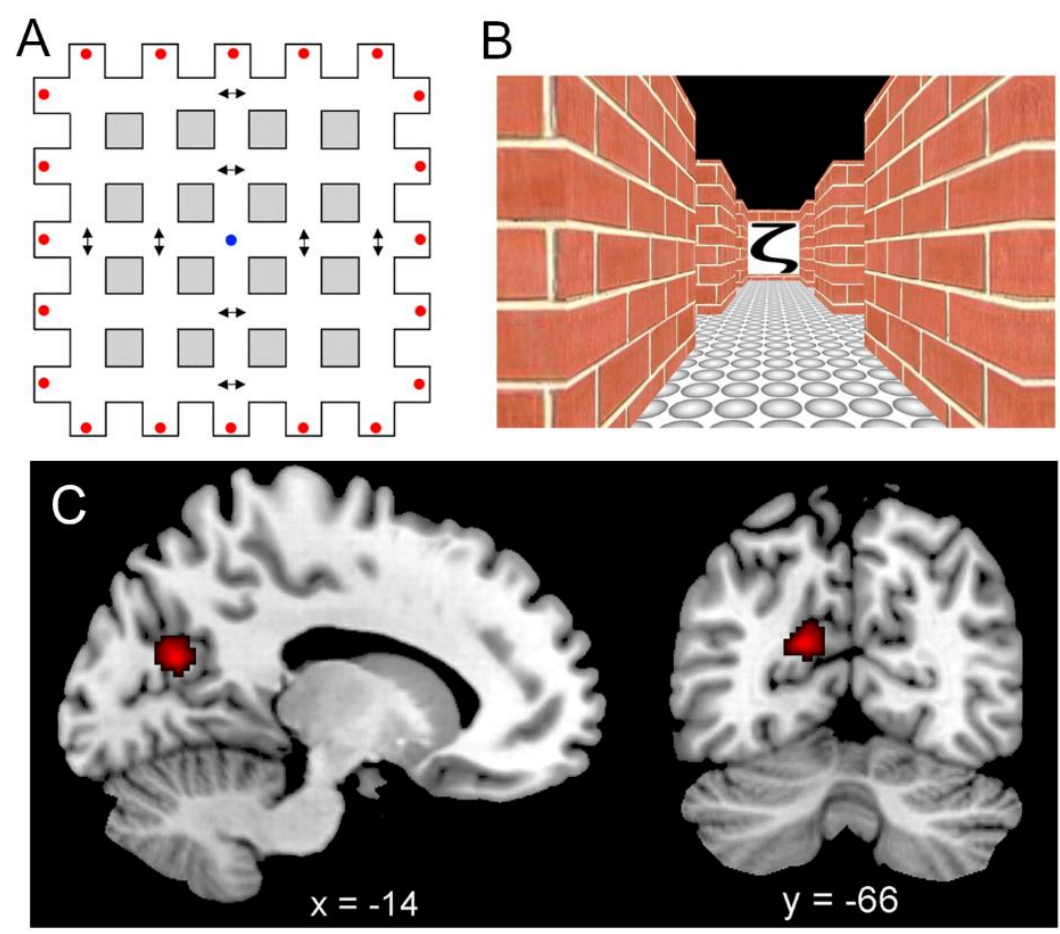

Figure 5. Schematics of the virtual environment and associated brain activity revealed through an analysis of neural adaptation effects. (A) Layout of the virtual maze as viewed from above, 
showing the $5 \times 5$ arrangement of corridors and the locations of landmarks (red dots). The arrows indicate the vantage points from which participants judged their location within the virtual grid. (B) Example first-person view from within the virtual environment, showing a pictorial landmark at the end of a corridor. (C) MRI brain slices showing heading-directionselective activity in the retrosplenial complex. Figure adapted from Baumann \& Mattingley (2010) under a Creative Commons Attribution 4.0 International (CC BY 4.0) license.

The suggestion of a role for the retrosplenial complex in spatial navigation is also consistent with observations that this region exhibits higher levels of neural activity for visual objects that typically serve as stable landmarks. For example, Auger and colleagues (2012) generated a large set of images depicting everyday outdoor items, and had a group of participants provide ratings along dimensions that included size, salience and permanence (i.e. how often an object could be expected to change position in everyday life). In the second part of the study, performed in the scanner, a new group of participants viewed an optimized subset of the original images while performing an orthogonal task (detecting the appearance of an irrelevant blue dot in the display). Performing a whole brain regression analysis with the factors of size and permanence, they found that the retrosplenial complex responded significantly more strongly to objects that were more highly rated on permanence, whereas the same region showed no differential response to changes in the size of the objects. In contrast, activity in the parahippocampal gyrus increased only moderately with the permanence of objects and showed a similarly modest response to changes in object size. Importantly, the task used by Auger et al. (2012) did not explicitly require participants to judge whether the objects could be considered stable landmarks, suggesting that activity in the 
retrosplenial cortex reflects implicit, experience-based knowledge concerning objects that can be used as reliable directional anchors. Along similar lines, Park and colleagues (2015) observed that fMRI signals in the retrosplenial complex also scale with the size of scenes, with higher levels of activity in response to larger environments. One interpretation of this finding is that the retrosplenial complex learned to recognise larger environments as being more navigationally relevant (analogous to larger and more stable landmarks). The sensitivity of the retrosplenial complex to egocentric distance was confirmed in a similar fMRI study by Persichetti and Dilks (2016). In their study, however, only changes in distance were investigated rather than specific directional relationships between distance and the strength of neural activation.

In addition to its involvement in representing directional cues, the retrosplenial complex also appears to play a more general role in the encoding, consolidation and retrieval of long-term spatial knowledge. Early neuroimaging findings reported by Wolbers and Buechel (2005) suggested a role for the retrosplenial complex in spatial learning, as indicated by a positive correlation between $\mathrm{fMRI}$ activity in this region and expertise in a topographic learning task. Over six testing sessions participants were moved virtually through a complex hexagonal environment that contained houses as landmarks. Participants' spatial knowledge was assessed after each learning session by probing their knowledge of the positions of landmarks relative to one another. Specifically, participants were shown a single viewpoint and had to indicate the direction of a target landmark, which could be located to the left, right or behind their current location. Learning over the course of the experiment was accompanied by increases in retrosplenial complex activity, suggesting that this region is responsible for generating an increasingly complex (and potentially more veridical) representation of the external environment. These performance-related increases in retrosplenial cortex activity 
were also accompanied by learning-related increases in activity in the hippocampus. Interestingly, in contrast to retrosplenial complex activity, which reflected increasing expertise over the duration of the experiment, activity in the hippocampus was most prominent during the initial learning phase and diminished after performance had reached ceiling.

One interpretation of these results is that the retrosplenial complex, in line with its role in encoding directions, serves to integrate different egocentric viewpoints from the local environment into a coherent, global representation. With prolonged exposure and learning, this retrosplenial representation increases in complexity, as reflected by a monotonic increase in neural activity. In contrast, the hypothesized function of the hippocampus is to integrate the current learning experience into an existing representation of the environment. As learning progresses the number of new associations to be formed decreases, and this is reflected in weaker hippocampal activity at later stages of the learning process. A subsequent study by laria and colleagues (2007) employed a similar virtual navigation paradigm and also found learning-related activity in the retrosplenial complex and hippocampus. In contrast to the Wolbers and Buechel (2005) study, however, laria and colleagues (2007) found that hippocampal activity persisted throughout the entire learning phase, perhaps due to the use of random starting locations which in turn might have posed higher demands on the hippocampus.

The proposal that the retrosplenial complex integrates information across multiple egocentric views is also supported by the work of Park and Chun (2009). They showed participants sections of panoramic scenes, either in their order of appearance or randomized. Measuring the magnitude of adaptation in the $\mathrm{FMRI}$ signal for these two conditions, they found that 
activity in the retrosplenial complex decreased significantly for continuous sequences of panoramic images but remained unchanged when the same images were presented in random order. Interestingly, this pattern was not evident in parahippocampal cortex, suggesting that its representations are viewpoint specific. Robertson and colleagues (2016) employed a similar task, with overlapping and non-overlapping scene sequences, but now the scene images were taken from a $360^{\circ}$ panorama. Their $\mathrm{fMRI}$ results revealed the same pattern as described by Park and Chun (2009), indicating that the retrosplenial complex, but not the parahippocampal cortex, is sensitive to the contrast of overlapping vs. non-overlapping scenes.

Further support for the idea that the retrosplenial complex integrates environmental information across viewpoints comes from a study by Epstein and colleagues (2007). They used a different experimental paradigm in which participants viewed objects, as well as unfamiliar and familiar scenes (e.g., a university campus), and asked them to indicate either their familiarity with the scene, their orientation (e.g. "East or West facing?"), or their location (e.g. "West of East of $36^{\text {th }}$ Street?"). fMRI activity patterns in the parahippocampal cortex differed for objects and scenes but were not affected by the familiarity of the scene or the type of information requested in the judgments. In contrast, activity was significantly higher in the retrosplenial complex for familiar versus unfamiliar scenes, suggesting a role in memory encoding or retrieval. Activity in the retrosplenial complex increased further when participants were asked for heading information, and even more so when they were asked about the location of the vantage point. These findings are in line with the idea that the human retrosplenial cortex integrates individual egocentric viewpoints into a more holistic representation of the local environment. 
The notion that the retrosplenial complex is involved in the encoding of the locations of landmarks was corroborated in a recent study by Persichetti and Dilks (2019). Participants had to learn the locations of several buildings in a virtual environment, and fMRI was used to measure neural activity when participants retrieved relative directional information for pairs of buildings. Importantly, the buildings could be from the same category or from different categories (i.e. different instances of coffee shops, dentists, gyms, and hardware stores), or from the same or a different region of the virtual environment (i.e., NW, NE, SW, or SE). Applying a multidimensional scaling approach to the fMRI data, they identified a functional double-dissociation. Specifically, they found that the parahippocampal cortex was sensitive to building category, but not to location, whereas the retrosplenial complex was sensitive to location but not to building category. In contrast to these findings, Vass and Epstein (2013) reported location-specific signals in both the retrosplenial complex and the parahippocampal cortex. Importantly, however, Vass and Epstein (2013) used photographs of real buildings rather than virtual images, raising the possibility that the variable of 'location' may not have been as salient as that yielded by the virtual environment employed by Persichetti \& Dilks (2019). Nevertheless, taken together the results of these investigations further support the hypothesis that the retrosplenial complex is fundamentally involved in the spatial mapping of landmarks rather than in the analysis of specific visuospatial properties of landmarks.

Further evidence that the retrosplenial complex represents the local environment on a more holistic and abstract level than the parahippocampal cortex comes from an fMRI study that investigated how the relative size of physical environments affects memory-related brain activity (Baumann \& Mattingley, 2013). In a two-stage design, participants were first trained outside the scanner to efficiently navigate in differently sized virtual environments (see Fig 6a). In a subsequent fMRI stage, participants were shown landmarks from the different 
environments and were asked to classify their origin. The rationale was that the task should trigger neural representations of the corresponding environments, which could then be compared in terms of their neural activity profiles. The authors found that activity in the retrosplenial complex (as well as in the right posterior hippocampus), scaled positively with the size of the environment in which the landmark had been encountered. The fact that retrosplenial activity was modulated by the global geometric aspects of the environment suggests that this region, in concert with the right hippocampus, plays a crucial role in constructing and maintaining spatial representations of the environment.

A

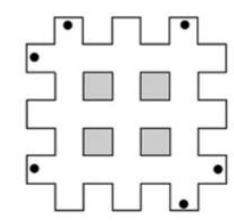

C

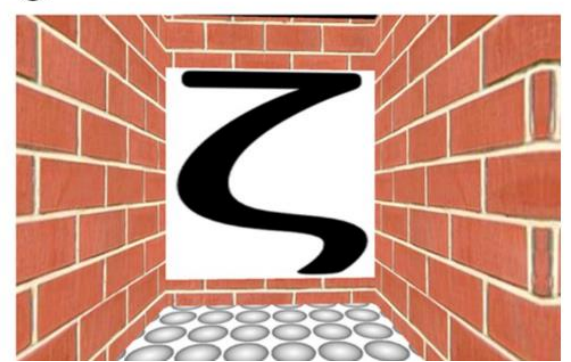

$\mathrm{B}$

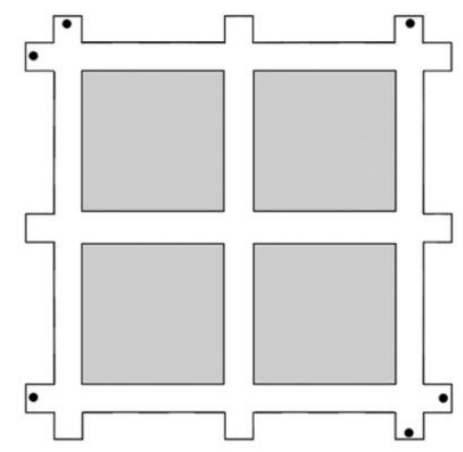

D

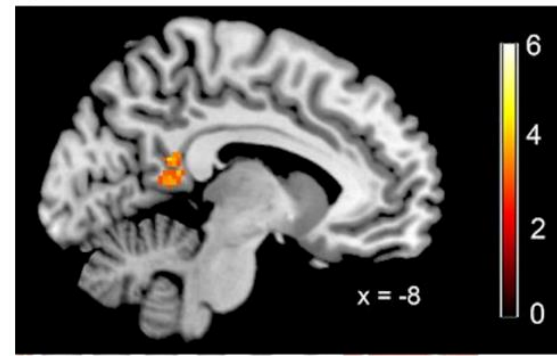

Figure 6. Schematics of the virtual environments and associated brain responses. Layout of the (A) small, and (B) large virtual mazes as viewed from above, showing the $3 \times 3$ arrangement of corridors and the locations of landmarks (black dots). (C) Example first-person view from within the virtual environment, showing a pictorial landmark at the end of a corridor. (D) Sagittal MR slices shows significant retrosplenial fMRI activity for the effect of 
maze size (Large > Small). Figure adapted from Baumann \& Mattingley (2013) under a

Creative Commons Attribution 4.0 International (CC BY 4.0) license.

\section{Dorsal Striatum}

The dorsal striatum is a large cluster of neurons in the basal ganglia and consists of the caudate nucleus and the putamen (see Fig 1 ). The dorsal striatum is widely recognized for its important contribution to motor control (Haber 2016), but it is also clear that it has a wider variety of functions, specifically in stimulus-response learning (Featherstone \& McDonald, 2004). Its relevance for spatial navigation was recognised more than 50 years ago, when it was found that lesions of the dorsal striatum in rats caused profound deficits in a radial maze navigation task (Potegal et al., 1969). Subsequent rodent experiments systematically compared striatal and hippocampal roles in spatial learning (e.g. Packard \& McGaugh 1992, 1996), and suggested that the hippocampus underpins environment-centred representations of location, whereas the dorsal striatum is linked to stimulus-response learning relative to individual landmarks. Those findings were mirrored by an fMRI study conducted by laria and colleagues (2003) which investigated human brain activity associated with spontaneous and flexible use of environment-centred and stimulus-response based navigation strategies. The experiment used a virtual environment and required participants to learn and later retrieve objects in a radial-arm maze. Critically, distal landmarks that were visible during the learning phase were removed during the retrieval phase. The rationale behind this manipulation was that participants who employed an environment-based learning strategy (i.e., using the spatial configuration provided by distal landmarks) should make more errors than participants who used a response-based strategy (i.e., effectively counting the corridors in the radial 
maze). As in the earlier rodent work, use of environment-centred strategies was found to be correlated with hippocampal activity, whereas use of response-based strategies correlated with activity in the caudate nucleus. In another relevant study, Bohbot and colleagues (2007) found that individuals with higher grey-matter density in the caudate nucleus were more likely to use single-landmark response-based strategies in a virtual environment, whereas those with higher grey matter density in the hippocampus tended to use navigation strategies that relied on participants learning the relationships between multiple landmarks.

Doeller and colleagues (2008) monitored brain activity in human participants for object locations that were encoded either relative to the circular boundary of a virtual environment, or relative to discrete landmarks within the same environment. They found that right posterior hippocampal activation was correlated with learning of boundary-related locations, whereas activity in the right dorsal striatum reflected learning of landmark-related locations. Using a similar design, Wegman and colleagues (2014) found that learning-related fMRI activity in the dorsal striatum was related to navigation strategies that relied on single landmarks, whereas strategies relating to configurations of landmarks were linked to modulations within the hippocampus. In separate work, it was shown that the striatal contribution to spatial navigation not only depends on task configurations, but also on task instructions. In their fMRI study, Baumann et al. (2012) had participants navigate a virtual environment with the goal of encoding the location of a target object relative to a single reference landmark (Fig 7a). Importantly, on a trial-by-trial basis, participants were either instructed to encode the distance between the target object and the reference landmark (the co-ordinate condition), or the quadrant of the arena, as indicated by the reference landmark, in which the target object was located (the categorical condition). Despite identical visual environment and movement patterns during the encoding period, the co-ordinate condition 
was associated with significantly higher fMRI activity in the dorsal striatum than the categorical condition (see Fig 7b). Taken together, these imaging studies strongly suggest that the distinction between hippocampal spatial (configurational) learning, and striatal responsebased learning, as originally identified in rodents (Packard \& McGaugh 1992, 1996), is also applicable in humans.
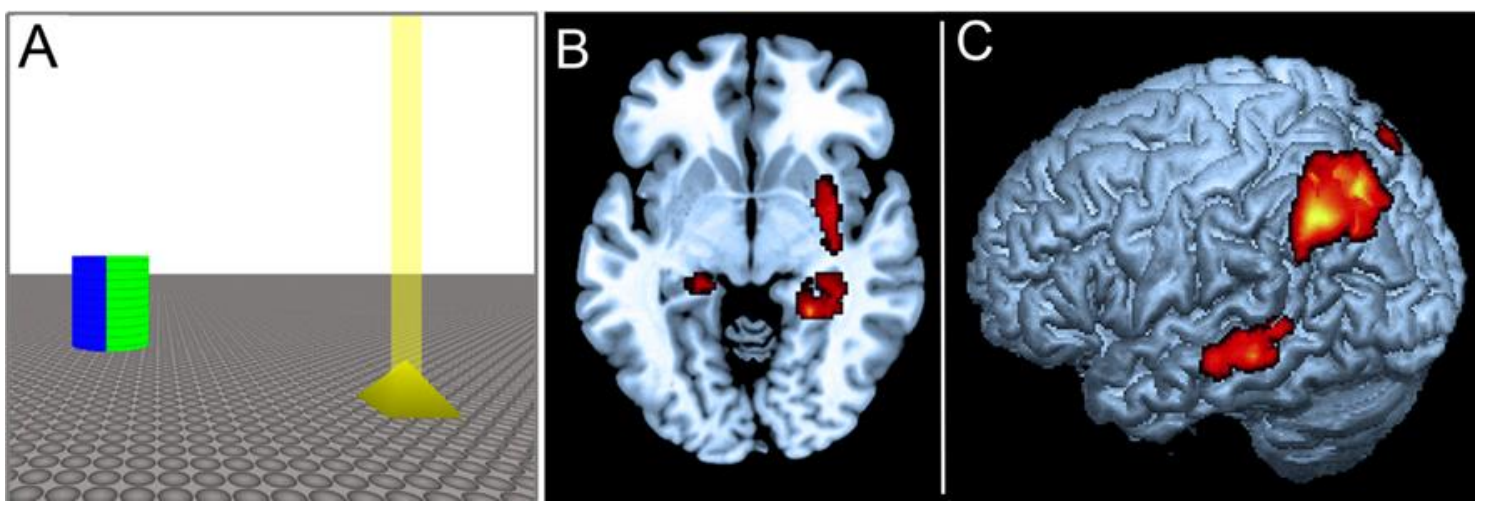

Figure 7. Schematic of the virtual environment used in the experiment by Baumann et al. (2012) and associated fMRI activity. (A) First-person view of the virtual environment. Participants were required to actively navigate the arena and to encode either the distance between the target object (yellow pyramid) and the multicoloured reference landmark (coordinate task), or the quadrant of the arena, as indicated by landmark, within which the target object was located (categorical task). (B) Axial brain slice showing greater right striatal and hippocampal activity during the encoding of co-ordinate (i.e. metrically precise) spatial relations. (C) Rendered image of the left hemisphere, showing greater left posterior parietal activity during the encoding of categorical spatial relations. Figure adapted from Baumann et al. (2012) https://doi.org/10.1016/j.neuroimage.2012.01.089, as permitted under the Elsevier publishing agreement. 
Human fMRI-based connectivity studies have revealed extensive functional connections between the dorsal striatum and the frontal cortex, but not between the striatum and medial temporal structures (Choi et al., 2012; Di Martino et al. 2008). This suggests that striatal and hippocampal learning circuits can operate independently, which is in line with observations of analogous functional dissociations in rodent lesion studies (e.g. Packard \& McGaugh 1992, 1996). In humans, white matter densities of the dorsal striatum and hippocampus are inversely correlated (Bohbot et al. 2007), which lends further support to the idea that independent and complementary learning mechanisms are supported by these two structures. However, more recent human functional imaging studies have found that in highly familiar environments with overlapping spatial routes (i.e. paths that connect more than two locations), co-operative interactions between the striatum and hippocampus underlie successful navigation performance (Brown et al., 2012; Brown and Stern, 2014).

For example, Brown and colleagues (2012) first trained participants outside the scanner to navigate several routes through a maze of hallways. Importantly, some of the routes overlapped (i.e. certain hallway segments were used by more than one route), whereas in the 'non-overlapping' condition the routes did not share any hallway segments. In the second phase of the study, in which brain activity was measured with fMRI, participants were passively moved through the hallways along previously learned routes. At certain choice points (intersections) participants were asked to indicate via button-press which direction to take to reach a specified goal location. Brown et al. (2012) found that the hippocampus and dorsal striatum (specifically the caudate nucleus) both exhibited increased indirect connectivity via the orbitofrontal cortex whenever participants made successful navigational 
decisions in trials involving overlapping path trajectories. This finding suggests that the orbitofrontal cortex acts as an interface for mediating cooperative interactions between hippocampal and striatal memory systems when people navigate environments that require contextual information to distinguish an intended path from other potential paths, thus enabling the navigator to resolve ambiguities. A follow-up study, using a similar environment and task, found increased parahippocampal cortex activity associated with learning of novel spatial cues (i.e. views of unique start locations) that allowed participants to distinguish between overlapping routes (Brown \& Stern, 2014). Taken together, the human imaging findings suggest that integration of response-based striatal codes, with allocentric hippocampal representations, and contextual cues encoded by the parahippocampal cortex, allow for context-based, flexible, and ultimately accurate navigational decision-making.

\section{Posterior Parietal Cortex}

The posterior parietal cortex in humans consists of BA5, BA7, BA39, and BA40 (Whitlock, 2017; see Fig 1). This region is reciprocally connected with the retrosplenial cortex/complex, and via this structure is also connected indirectly with the hippocampus (Witter, 1993). The posterior parietal cortex is thus anatomically ideally situated to play a role in spatial navigation. Based on monkey studies, an early hypothesis situated the role of the posterior parietal cortex in navigation into the broader framework of dual higher-order visual processing streams (Mishkin et al., 1983). According to this framework, a dorsal pathway connecting the primary visual cortex with the posterior parietal lobe is specialised for processing the locations of objects in space (i.e. the so-called "where" stream). By contrast, a ventral processing pathway connecting the primary visual cortex with the temporal lobe is concerned with extracting the detailed features of objects (i.e. the so-called "what" stream; 
Mishkin et al., 1983). Later findings, derived from human neuropsychological studies of the neglect syndrome (a condition in which patients fail to attend or respond to stimuli on one side of space), indicated a role for the posterior parietal cortex in integrating visual and sensorimotor signals for the coordination of visually guided reaching (Husain, 2008).

In contrast to the allocentric (i.e. environment-centred) hippocampal representation of space, the posterior parietal cortex is thought to provide an egocentric (i.e. body centred) reference frame for sensory and motoric spatial vectors (Stein, 1992). For instance, using fMRI Neggers and colleagues (2006) showed that activity in the superior parietal lobule increased when participants were asked to make egocentric spatial judgments (i.e. relative to their body), but not during allocentric spatial judgments (i.e. relative to an external visual stimulus). In regard to spatial navigation, it has been shown that patients with lesions of the posterior parietal cortex have markedly impaired egocentric route navigation even for well-learned environments and paths (Ciaramelli et al., 2010). These results have been corroborated by a neuropsychological study that assessed navigation performance using a virtual environment (Weniger et al., 2009). Despite continued interest in the neglect syndrome (Molenberghs et al. 2012), as mentioned above, the potential contributions of the posterior parietal cortex to spatial navigation have received relatively less attention from the brain imaging community than other structures. Although fMRI studies have identified navigation-related activity in the posterior parietal cortex (e.g., comparing active navigation to locomotion without spatial learning demands; Baumann et al. 2010; Groen et al. 2000), this brain area has rarely been the focus of navigation-specific investigations, and activation there has often been treated as incidental, or else has been interpreted in line with egocentric coding theory (Andersen and Buneo, 2002; Snyder et al., 1998). 
Nevertheless, a small number of studies have investigated the role of the parietal cortex in spatial navigation. One study used a virtual environment to characterize acquisition of pure route knowledge (i.e. encoding of consecutive landmark views from a ground-level perspective; Wolbers et al., 2004). Interestingly, this study indicated that the posterior portion of the inferior parietal cortex was the only region that showed increased activity in association with better behavioural performance, in contrast with retrosplenial and medial temporal regions which showed no such relationship. Another fMRI study by Spiers and Maguire (2007), who used a taxi-driver task in a virtual city, identified an added facet of parietal involvement in egocentric navigation. They found that activity in bilateral posterior parietal cortex was significantly correlated with egocentric angular direction to the goal specifically, higher activity was associated with turning away from the goal - suggesting that parietal cortex encodes a directional error signal during navigation. Interestingly, no such relationship was observed in either the medial temporal region or retrosplenial cortex.

Relevant work in rodents has also suggested a role for the posterior parietal cortex in navigation behaviour. Goodrich-Hunsaker and colleagues (2005) employed a noveltydetection task in which rats were initially familiarized with a small number of simple geometric landmarks in a circular arena. In a second stage, the investigators altered either the categorical relationships between the landmarks (i.e. their general spatial layout) or their coordinate relationships (i.e. the distances between landmarks). Goodrich-Hunsaker and colleagues found that rats with parietal lesions showed impairments specifically in recognising changes in the categorical relationships between objects, whereas those with hippocampal lesions exhibited deficits in recognising coordinate changes among the landmarks. Baumann et al. (2012) adapted this task for use in humans, as described earlier. Recall that these authors developed a sparse virtual-reality environment and instructed 
participants to encode either the coordinate or categorical location of a target object relative to a single reference landmark (see Fig 7a). They observed greater fMRI activity in the posterior parietal cortex on trials in which participants were required to encode the categorical relationship between the target and the reference landmark (see Figure 7c), and greater hippocampal and striatal activity on trials in which participants were required to encode the metric distance between the target object and the landmark (see Fig 7b).

Another example of a non-traditional parietal contribution to spatial navigation comes from the work of Nitz (2006), who recorded from rat parietal neurons during active navigation in a maze. Nitz (2006) found that parietal neurons showed selective firing for certain subsections within a larger route. Unlike hippocampal neurons, which also fire selectively to subsections within a route, the firing of parietal neurons did not depend on the animal's allocentric location within the surrounding environment, but only on its relative position within the geometric structure of the route.

In another human study, Howard and colleagues (2013) investigated the role of parietal cortex in encoding of natural scenes and object-background relationships. They used an incidental target detection test to identify patterns of brain activity related to spatial changes in natural scenes. As shown in Figure 8a, participants viewed a series of images consisting of object-scene pairs, and each object-scene pair was shown twice. Importantly, for the second presentation the spatial relationship between object and scene was manipulated to create different experimental conditions. Key conditions involved either no spatial change, an object position change, a scene position change, an object plus scene position change, or a completely novel scene (see Figure 8a). Only the superior parietal lobule was sensitive to changes in the spatial arrangement of scenes, i.e. not the scene novelty per se (see Figure 
$8 b, c)$. Given that the experimental task did not require participants to attend or react to scene changes, the findings suggest that the posterior parietal cortex contributes to spatial coding of the visuo-spatial environment without requiring conscious awareness. Interestingly, the response profile of the superior parietal lobule to changes in object-scene relationships is similar to that observed in the hippocampus (Howard et al., 2011), suggesting a functional relationship.

Another imaging study by van Assche and colleagues (2016) also supports a role for the posterior parietal cortex in the processing of scenes. They presented participants with sequences of images of a common scene from four different viewpoints and found that parietal activity increased with the number of scenes displayed within the sequence, suggesting that the parietal cortex integrates scene information across viewpoints. As in the study of Howard and colleagues (2013), participants were engaged in an orthogonal task during the imaging protocol (judging whether the scene was 'lively' or 'calm'), again suggesting that parietal activity was not dependent on explicit encoding or retrieval processes. These imaging findings are consistent with reports that purely imagined target directions can be decoded from fMRI activity patterns (Schindler \& Bartels, 2013). 


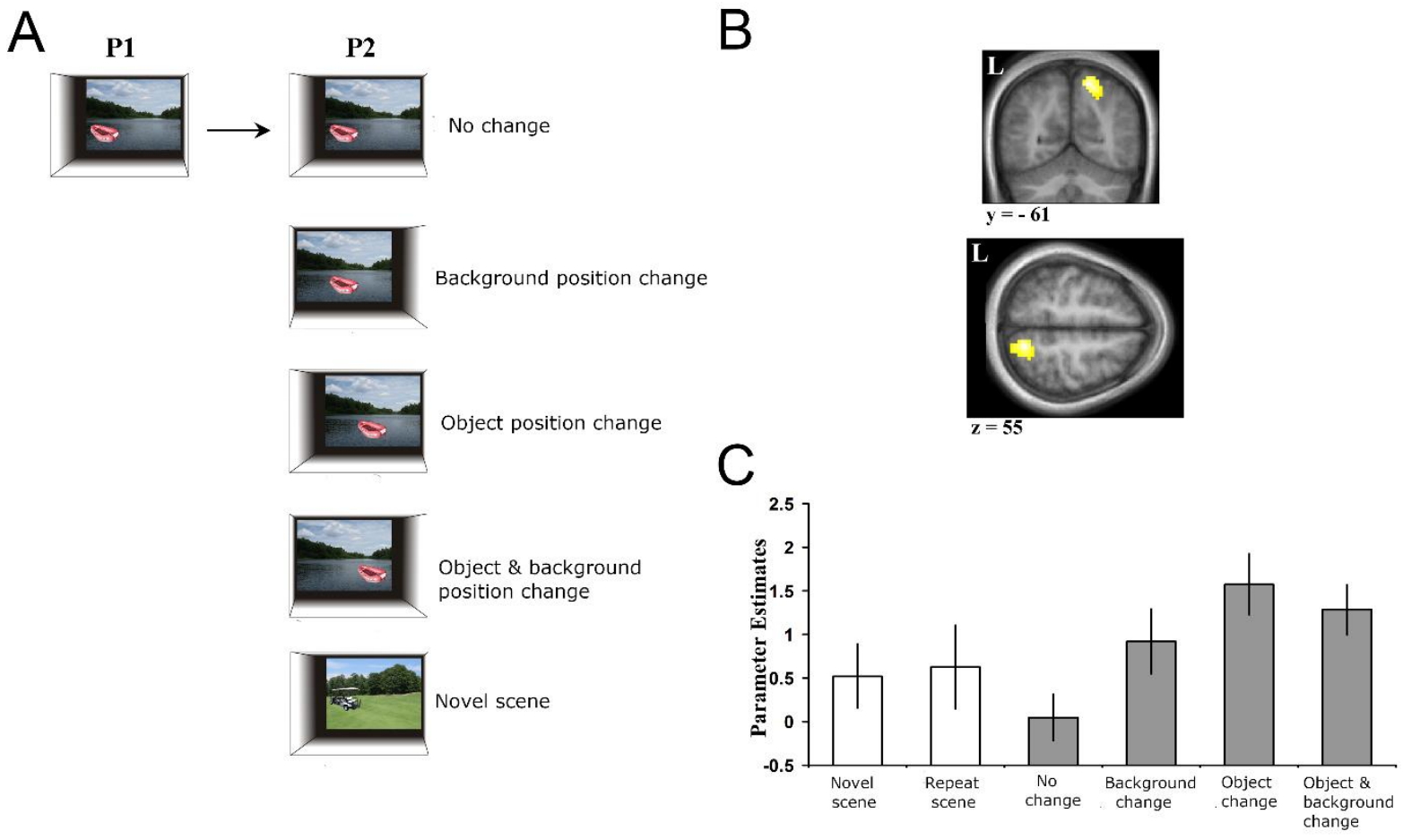

Figure 8. Experimental conditions and brain activations in the study by Howard et al. (2013).

(A) During the second presentation the spatial relations between objects and backgrounds were changed to create six experimental conditions, as shown. (B) Increased fMRI activity in the superior parietal lobule in response to the three types of spatial change relative to no change. (C) Peak-voxel parameter estimates for the six conditions. Figure adapted from Howard et al. (2013). Creative Commons Attribution 4.0 International (CC BY 4.0) license.

Taken together, the studies considered here suggest that the posterior parietal cortex plays a role in spatial navigation that goes beyond maintaining an egocentric spatial reference frame. Human neuroimaging and rodent electrophysiological findings suggest that the parietal cortex is involved in the allocentric coding of space. But this role is different from that of the hippocampus, in the sense it uses spatial representations that are less map-like, less precise and less cohesive, than those stored in the hippocampus. Notably, functional 
connectivity between the posterior parietal cortex and hippocampus has been found to increase if tasks require flexible rather than rigid forms of allocentric retrieval (i.e. requiring extrapolation of positional information across several earlier trials; Zhang \& Ekstrom, 2013). More work will be needed to fully understand how the posterior parietal cortex contributes to spatial navigation.

\section{Limitations}

As noted earlier, despite its unique advantages for studying spatial navigation, non-invasive brain imaging also has its weaknesses. Arguably the biggest disadvantage with $\mathrm{fMRI}$ is that it requires the immobilisation of the participant's head during image acquisition. This makes it impossible to record brain signals during actual navigation, unlike rodent electrophysiological studies which can employ wireless recording techniques (e.g. Fan et al. 2011). Whereas the fidelity of the visual component of simulated navigation experiences is typically acceptable, other important components related to self-motion, such as motoric, vestibular, and proprioceptive signals, are lacking (for an overview, see Taube et al. 2013). These shortcomings are unlikely to be resolved in the near future, but there are several creative workarounds that can be employed. One approach is to use active, real-life encoding conditions, and then conduct the retrieval task in the scanner, for example by using snapshots of stimuli from the learned environment. This approach allows the investigation of retrievalrelated activity for environments that have been learned under ecologically valid conditions. For instance, Rosenbaum et al. (2004) and Patai et al. (2019) used this approach to reveal a role for the retrosplenial complex in the long-term consolidation of environments. In addition, it should be possible to systematically manipulate self-movement related signals during encoding (i.e. active vs. passive navigation) and subsequently to measure their effect on 
retrieval-related fMRI activity. Further extending this approach, both encoding and retrieval sessions could involve physical navigation (either in the real-world or under virtual reality), and $\mathrm{fMRI}$ would be employed solely to probe maintenance-related activity. This would allow investigation of predictive relationships between encoding performance, retrieval performance and maintenance-related neural activity and connectivity patterns. Once again, the amount of self-motion required during encoding and retrieval could be systematically varied and its effect on maintenance-related fMRI activity analysed.

\section{Conclusion}

In the preceding sections we reviewed examples of how human neuroimaging data have expanded our understanding of extrahippocampal contributions to spatial navigation, by corroborating and extending earlier rodent electrophysiological and lesion studies, as well as human neuropsychological case studies. Importantly, neuroimaging with fMRI offers finegrained (millimetre scale) function-location mapping, and even more precise (sub-millimetre) anatomical mapping. These fMRI studies have revealed that whereas the hippocampus plays a primary role in encoding and maintaining allocentric representations of the environment, the parahippocampal cortex provides important pre-processing of visual-spatial layouts and contextual cues. The retrosplenial complex provides heading-related inputs to the hippocampus, which are anchored in proximal as well as distal allocentric reference frames, but also plays a role in representing salient stable landmarks, and in the maintenance of longterm spatial knowledge. The dorsal striatum provides a response-based implicit learning mechanism that complements hippocampal allocentric representations. Finally, the posterior parietal cortex is involved in providing body-centred reference frames for the guidance of actions, but may play additional roles including the encoding of categorical as well as route- 
based spatial representations. A key message is that all these regions must interact functionally to regulate successful navigational behaviour.

Importantly, fMRI affords the measurement of whole-brain inter-regional functional and structural connectivity and can thereby provide further insights into the functional contributions of extrahippocampal areas to spatial navigation. A recent resting-state fMRI study in humans (Boccia et al., 2017) revealed functional connections between the hippocampus, parahippocampal cortex, retrosplenial complex, and posterior parietal cortex (see Fig 9), suggesting a functional network in support of spatial navigation (Kravitz et al. 2011b).

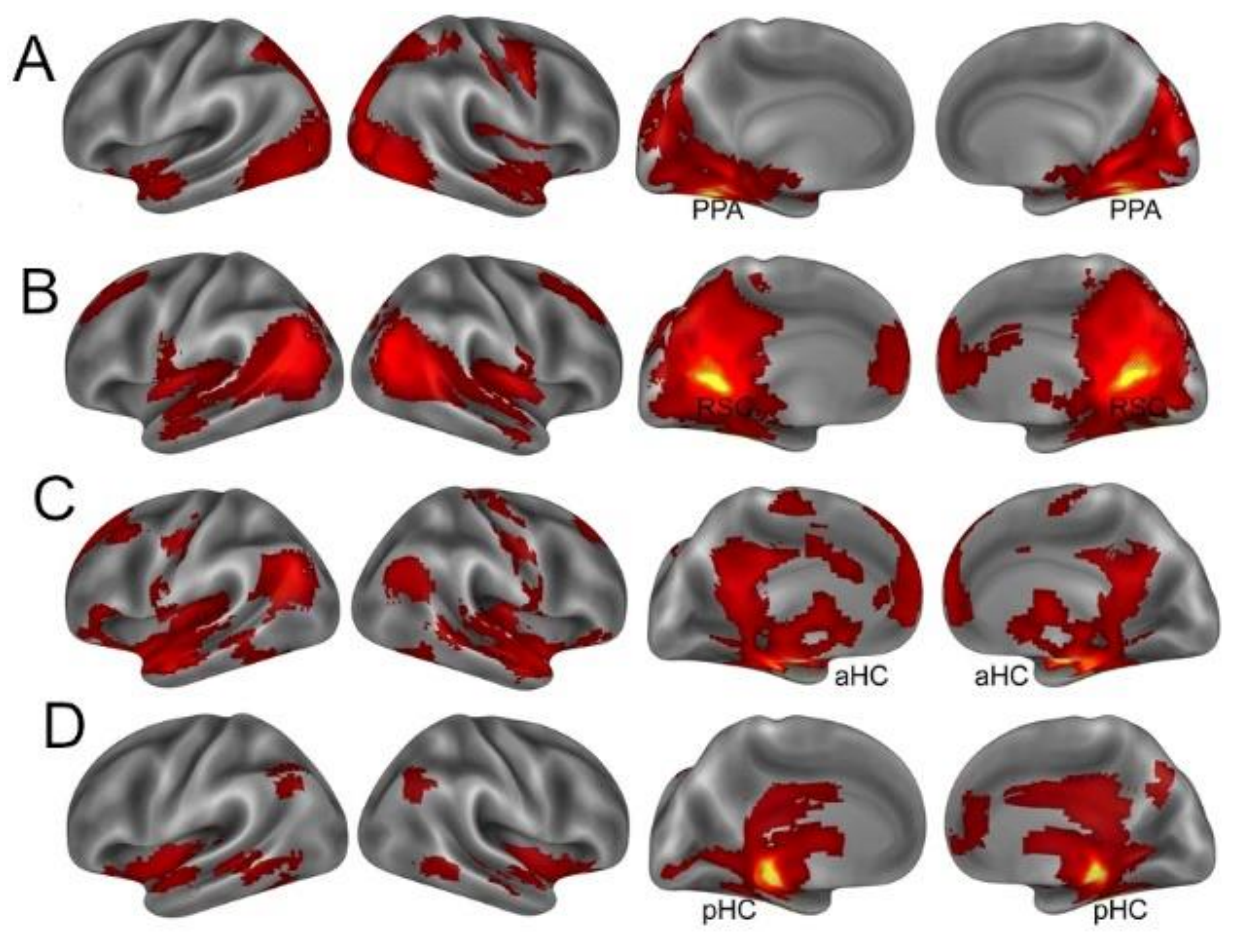

Figure 9. Functional whole-brain connectivity patterns for different seed regions (red denotes higher levels of connectivity). (A) Parahippocampal cortex seed. (B) Retrosplenial complex seed. (C) Anterior hippocampus seed. (D) Posterior hippocampus seed. Figure adapted with permission from Boccia et al. (2017). 
The dorsal striatum, however, appears to be only indirectly connected to this network, with links to the hippocampus via the frontal cortex and the ventral striatal reward circuit (Goodroe et al. 2018; see also Fig 1). This corresponds with the proposed balancing and competitive role of the dorsal striatum in relation to the network described above encompassing the hippocampus, parahippocampal cortex, retrosplenial complex and posterior parietal cortex. To further advance understanding of the human navigation system it will be necessary to move beyond simple correlational analyses and to systematically test mechanistic models using techniques such as dynamic causal modelling (Friston et al., 2003, 2019). This method allows quantitative comparison of competing models of task-dependent functional interactions between pre-specified brain areas. Although this approach has been successfully applied to questions relating to spatial navigation, to date the work has focused on mini networks, consisting of only two interconnected areas, such as between the hippocampus and parahippocampal cortex (Auger \& Maguire, 2018), or between the retrosplenial and medial temporal areas (Chadwick et al. 2013). Future studies should aim to investigate functional causal networks that include the hippocampus as well as the four extrahippocampal regions discussed in this review.

In conclusion, neuroimaging complements other neuroscientific techniques in the investigation of spatial navigation and its underlying hippocampal and extrahippocampal structures. The development of creative experimental designs in combination with technological advances will continue to provide new knowledge in this field and help to answer new research questions. 


\section{Acknowledgements}

This work was supported by the Australian Research Council (ARC) Centre of Excellence for Integrative Brain Function (ARC Centre Grant CE140100007). 


\section{References}

Abrahams, S., Pickering, A., Polkey, C. E., \& Morris, R. G. (1997). Spatial memory deficits in patients with unilateral damage to the right hippocampal formation. Neuropsychologia, 35(1), 11-24. https://doi.org/10.1016/s0028-3932(96)00051-6

Aguirre, G. K., Detre, J. A., Alsop, D. C., \& D'Esposito, M. (1996). The parahippocampus subserves topographical learning in man. Cerebral cortex, 6(6), 823-829.

https://doi.org/10.1093/cercor/6.6.823

Aminoff, E. M., Kveraga, K., \& Bar, M. (2013). The role of the parahippocampal cortex in cognition. Trends in cognitive sciences, 17(8), 379-390.

https://doi.org/10.1016/j.tics.2013.06.009

Andersen, R. A., \& Buneo, C. A. (2002). Intentional maps in posterior parietal cortex. Annual review of neuroscience, 25, 189-220.

https://doi.org/10.1146/annurev.neuro.25.112701.142922

Becker, S., \&Burgess, N. (2001). A model of spatial recall, mental imagery and neglect.

Neural information processing systems, 13, 96-102

van Assche, M., Kebets, V., Vuilleumier, P., \& Assal, F. (2016). Functional Dissociations Within Posterior Parietal Cortex During Scene Integration and Viewpoint Changes. Cerebral cortex (New York, N.Y. : 1991), 26(2), 586-598. https://doi.org/10.1093/cercor/bhu215 
Auger, S. D., \& Maguire, E. A. (2018). Retrosplenial Cortex Indexes Stability beyond the Spatial Domain. The Journal of neuroscience : the official journal of the Society for Neuroscience, 38(6), 1472-1481. https://doi.org/10.1523/JNEUROSCI.2602-17.2017

Auger, S. D., Mullally, S. L., \& Maguire, E. A. (2012). Retrosplenial cortex codes for permanent landmarks. PloS one, 7(8), e43620.

https://doi.org/10.1371/journal.pone.0043620

Baldassano C, Beck DM, Fei-Fei L (2013) Differential connectivity within the parahippocampal place area. Neuroimage 75:228-237, doi:10.1016/j.neuroimage.2013.02.073, pmid:23507385

Bar, M., \& Aminoff, E. (2003). Cortical analysis of visual context. Neuron, 38(2), 347-358. https://doi.org/10.1016/s0896-6273(03)00167-3

Baumann, O., Chan, E., \& Mattingley, J. B. (2010). Dissociable neural circuits for encoding and retrieval of object locations during active navigation in humans. Neurolmage, 49(3), 2816-2825. https://doi.org/10.1016/j.neuroimage.2009.10.021

Baumann, O., Chan, E., \& Mattingley, J. B. (2012). Distinct neural networks underlie encoding of categorical versus coordinate spatial relations during active navigation. Neurolmage, 60(3), 1630-1637. https://doi.org/10.1016/j.neuroimage.2012.01.089 Baumann, O., \& Mattingley, J. B. (2010). Medial parietal cortex encodes perceived heading direction in humans. The Journal of neuroscience, 30(39), 12897-12901. https://doi.org/10.1523/JNEUROSCI.3077-10.2010 
Baumann, O., \& Mattingley, J. B. (2013). Dissociable representations of environmental size and complexity in the human hippocampus. The Journal of neuroscience, 33(25), 1052610533. doi:10.1523/JNEUROSCI.0350-13.2013

Baumann, O., \& Mattingley, J. B. (2016). Functional Organization of the Parahippocampal Cortex: Dissociable Roles for Context Representations and the Perception of Visual Scenes. The Journal of neuroscience : the official journal of the Society for Neuroscience, 36(8), 2536-2542. https://doi.org/10.1523/JNEUROSCI.3368-15.2016

Boccia, M., Sulpizio, V., Nemmi, F., Guariglia, C., \& Galati, G. (2017). Direct and indirect parieto-medial temporal pathways for spatial navigation in humans: evidence from restingstate functional connectivity. Brain structure \& function, 222(4), 1945-1957. https://doi.org/10.1007/s00429-016-1318-6

Bohbot, V. D., Kalina, M., Stepankova, K., Spackova, N., Petrides, M., \& Nadel, L. (1998). Spatial memory deficits in patients with lesions to the right hippocampus and to the right parahippocampal cortex. Neuropsychologia, 36(11), 1217-1238.

\section{https://doi.org/10.1016/s0028-3932(97)00161-9}

Bohbot, V. D., Lerch, J., Thorndycraft, B., laria, G., \& Zijdenbos, A. P. (2007). Gray matter differences correlate with spontaneous strategies in a human virtual navigation task. The Journal of neuroscience : the official journal of the Society for Neuroscience, 27(38), 1007810083. https://doi.org/10.1523/JNEUROSCI.1763-07.2007

Burgess N. (2014). The 2014 Nobel Prize in Physiology or Medicine: a spatial model for cognitive neuroscience. Neuron, 84(6), 1120-1125.

https://doi.org/10.1016/j.neuron.2014.12.009 
Brown, T. I., Ross, R. S., Tobyne, S. M., \& Stern, C. E. (2012). Cooperative interactions

between hippocampal and striatal systems support flexible navigation. Neurolmage, 60(2),

1316-1330. https://doi.org/10.1016/j.neuroimage.2012.01.046

Brown, T. I., \& Stern, C. E. (2014). Contributions of medial temporal lobe and striatal memory systems to learning and retrieving overlapping spatial memories. Cerebral Cortex, 24(7), 1906-1922. doi:10.1093/cercor/bht041

Chadwick, M. J., Mullally, S. L., \& Maguire, E. A. (2013). The hippocampus extrapolates beyond the view in scenes: an fMRI study of boundary extension. Cortex; a journal devoted to the study of the nervous system and behavior, 49(8), 2067-2079. https://doi.org/10.1016/j.cortex.2012.11.010

Chan, E., Baumann, O., Bellgrove, M. A., \& Mattingley, J. B. (2014). Negative emotional experiences during navigation enhance parahippocampal activity during recall of place information. Journal of cognitive neuroscience, 26(1), 154-164.

https://doi.org/10.1162/jocn_a_00468

Choi, E. Y., Yeo, B. T., \& Buckner, R. L. (2012). The organization of the human striatum estimated by intrinsic functional connectivity. Journal of neurophysiology, 108(8), 22422263. https://doi.org/10.1152/jn.00270.2012

Chrastil E. R. (2013). Neural evidence supports a novel framework for spatial navigation. Psychonomic bulletin \& review, 20(2), 208-227. https://doi.org/10.3758/s13423-012-0351-6 Ciaramelli, E., Rosenbaum, R. S., Solcz, S., Levine, B., \& Moscovitch, M. (2010). Mental space travel: damage to posterior parietal cortex prevents egocentric navigation and 
reexperiencing of remote spatial memories. Journal of experimental psychology. Learning, memory, and cognition, 36(3), 619-634. https://doi.org/10.1037/a0019181

Clark, R. E., \& Squire, L. R. (2013). Similarity in form and function of the hippocampus in rodents, monkeys, and humans. Proceedings of the National Academy of Sciences of the United States of America, 110(2), 10365-10370. https://doi.org/10.1073/pnas.1301225110 Di Martino, A., Scheres, A., Margulies, D. S., Kelly, A. M., Uddin, L. Q., Shehzad, Z., Biswal, B., Walters, J. R., Castellanos, F. X., \& Milham, M. P. (2008). Functional connectivity of human striatum: a resting state FMRI study. Cerebral cortex, 18(12), 2735-2747. https://doi.org/10.1093/cercor/bhn041

Doeller, C. F., King, J. A., \& Burgess, N. (2008). Parallel striatal and hippocampal systems for landmarks and boundaries in spatial memory. Proceedings of the National Academy of Sciences of the United States of America, 105(15), 5915-5920. https://doi.org/10.1073/pnas.0801489105

Epstein R. A. (2008). Parahippocampal and retrosplenial contributions to human spatial navigation. Trends in cognitive sciences, 12(10), 388-396.

https://doi.org/10.1016/j.tics.2008.07.004

Epstein, R., Harris, A., Stanley, D., \& Kanwisher, N. (1999). The parahippocampal place area: recognition, navigation, or encoding?. Neuron, 23(1), 115-125.

https://doi.org/10.1016/s0896-6273(00)80758-8

Epstein, R., \& Kanwisher, N. (1998). A cortical representation of the local visual environment. Nature, 392, 598-601 
Epstein, R. A., Parker, W. E., \& Feiler, A. M. (2007). Where am I now? Distinct roles for parahippocampal and retrosplenial cortices in place recognition. The Journal of neuroscience, 27(23), 6141-6149. https://doi.org/10.1523/JNEUROSCI.0799-07.2007

Epstein, R. A., \& Ward, E. J. (2010). How reliable are visual context effects in the parahippocampal place area?. Cerebral cortex (New York, N.Y. : 1991), 20(2), 294-303. https://doi.org/10.1093/cercor/bhp099

Fan, D., Rich, D., Holtzman, T., Ruther, P., Dalley, J. W., Lopez, A., Rossi, M. A., Barter, J. W., Salas-Meza, D., Herwik, S., Holzhammer, T., Morizio, J., \& Yin, H. H. (2011). A wireless multichannel recording system for freely behaving mice and rats. PloS one, 6(7), e22033. https://doi.org/10.1371/journal.pone.0022033

Featherstone, R. E., \& McDonald, R. J. (2004). Dorsal striatum and stimulus-response learning: lesions of the dorsolateral, but not dorsomedial, striatum impair acquisition of a simple discrimination task. Behavioural brain research, 150(1-2), 15-23.

https://doi.org/10.1016/S0166-4328(03)00218-3

Friston, K. J., Harrison, L., \& Penny, W. (2003). Dynamic causal

modelling. Neurolmage, 19(4), 1273-1302. https://doi.org/10.1016/s1053-8119(03)00202-7

Friston, K. J., Preller, K. H., Mathys, C., Cagnan, H., Heinzle, J., Razi, A., \& Zeidman, P. (2019).

Dynamic causal modelling revisited. Neurolmage, 199, 730-744.

https://doi.org/10.1016/j.neuroimage.2017.02.045

Ghaem, O., Mellet, E., Crivello, F., Tzourio, N., Mazoyer, B., Berthoz, A., \& Denis, M. (1997). Mental navigation along memorized routes activates the hippocampus, precuneus, and insula. Neuroreport, 8(3), 739-744. https://doi.org/10.1097/00001756-199702100-00032 
Goodroe, S. C., Starnes, J., \& Brown, T. I. (2018). The Complex Nature of Hippocampal-

Striatal Interactions in Spatial Navigation. Frontiers in human neuroscience, 12, 250.

https://doi.org/10.3389/fnhum.2018.00250

Goodrich-Hunsaker, N. J., Hunsaker, M. R., \& Kesner, R. P. (2005). Dissociating the role of the parietal cortex and dorsal hippocampus for spatial information processing. Behavioral neuroscience, 119(5), 1307-1315. https://doi.org/10.1037/0735-7044.119.5.1307

Grön, G., Wunderlich, A. P., Spitzer, M., Tomczak, R., \& Riepe, M. W. (2000). Brain activation during human navigation: gender-different neural networks as substrate of performance. Nature neuroscience, 3(4), 404-408. https://doi.org/10.1038/73980

Haber S. N. (2016). Corticostriatal circuitry. Dialogues in clinical neuroscience, 18(1), 7-21.

Husain M(2008), Scholarpedia, 3(2):3681. doi:10.4249/scholarpedia.3681

Howard, L. R., Kumaran, D., Ólafsdóttir, H. F., \& Spiers, H. J. (2011). Double dissociation between hippocampal and parahippocampal responses to object-background context and scene novelty. The Journal of neuroscience : the official journal of the Society for Neuroscience, 31(14), 5253-5261. https://doi.org/10.1523/JNEUROSCI.6055-10.2011 Howard, L. R., Kumaran, D., Ólafsdóttir, H. F., \& Spiers, H. J. (2013). Dissociation between dorsal and ventral posterior parietal cortical responses to incidental changes in natural scenes. PloS one, 8(7), e67988. https://doi.org/10.1371/journal.pone.0067988

Iaria, G., Chen, J. K., Guariglia, C., Ptito, A., \& Petrides, M. (2007). Retrosplenial and hippocampal brain regions in human navigation: complementary functional contributions to the formation and use of cognitive maps. The European journal of neuroscience, 25(3), 890899. https://doi.org/10.1111/j.1460-9568.2007.05371.x 
laria, G., Petrides, M., Dagher, A., Pike, B., \& Bohbot, V. D. (2003). Cognitive strategies

dependent on the hippocampus and caudate nucleus in human navigation: variability and change with practice. The Journal of neuroscience : the official journal of the Society for Neuroscience, 23(13), 5945-5952. https://doi.org/10.1523/JNEUROSCI.23-13-05945.2003

Janzen, G., \& van Turennout, M. (2004). Selective neural representation of objects relevant for navigation. Nature neuroscience, 7(6), 673-677. https://doi.org/10.1038/nn1257

Kemp, J. M., \& Powell, T. P. (1971). The connexions of the striatum and globus pallidus: synthesis and speculation. Philosophical transactions of the Royal Society of London. Series B, Biological sciences, 262(845), 441-457. https://doi.org/10.1098/rstb.1971.0106

Kim, M., \& Maguire, E. A. (2019). Encoding of 3D head direction information in the human brain. Hippocampus, 29(7), 619-629. https://doi.org/10.1002/hipo.23060

Kobayashi, Y., \& Amaral, D. G. (2003). Macaque monkey retrosplenial cortex: II. Cortical afferents. The Journal of comparative neurology, 466(1), 48-79.

https://doi.org/10.1002/cne.10883

Konkle, T., \& Oliva, A. (2012). A real-world size organization of object responses in occipitotemporal cortex. Neuron, 74(6), 1114-1124.

https://doi.org/10.1016/j.neuron.2012.04.036

Kornblith, S., Cheng, X., Ohayon, S., \& Tsao, D. Y. (2013). A network for scene processing in the macaque temporal lobe. Neuron, 79(4), 766-781.

https://doi.org/10.1016/j.neuron.2013.06.015

Kravitz, D. J., Peng, C. S., \& Baker, C. I. (2011a). Real-world scene representations in highlevel visual cortex: it's the spaces more than the places. The Journal of neuroscience : the 
official journal of the Society for Neuroscience, 31(20), 7322-7333.

https://doi.org/10.1523/JNEUROSCI.4588-10.2011

Kravitz, D. J., Saleem, K. S., Baker, C. I., \& Mishkin, M. (2011b). A new neural framework for visuospatial processing. Nature reviews. Neuroscience, 12(4), 217-230.

https://doi.org/10.1038/nrn3008

Lang, P.J., Bradley, M.M., Cuthbert, B.N., 1999. International affective picture system (IAPS:

Technical manual and affective ratings). Gainesville: University of Florida, Center for

Research in Psychophysiology.

LeVan, P., Akin, B., \& Hennig, J. (2018). Fast imaging for mapping dynamic networks.

Neurolmage, 180, 547-558. https://doi.org/10.1016/j.neuroimage.2017.08.029

Maguire, E. A., Frackowiak, R. S., \& Frith, C. D. (1996). Learning to find your way: a role for the human hippocampal formation. Proceedings. Biological sciences, 263(1377), 1745-1750. https://doi.org/10.1098/rspb.1996.0255

Maguire, E. A., Frackowiak, R. S., \& Frith, C. D. (1997). Recalling routes around london: activation of the right hippocampus in taxi drivers. The Journal of neuroscience, 17(18), 7103-7110. https://doi.org/10.1523/JNEUROSCI.17-18-07103.1997

Marchette, S. A., Vass, L. K., Ryan, J., \& Epstein, R. A. (2014). Anchoring the neural compass: coding of local spatial reference frames in human medial parietal lobe. Nature neuroscience, 17(11), 1598-1606. https://doi.org/10.1038/nn.3834

Marchette, S. A., Vass, L. K., Ryan, J., \& Epstein, R. A. (2015). Outside Looking In: Landmark Generalization in the Human Navigational System. The Journal of neuroscience, 35(44), 14896-14908. https://doi.org/10.1523/JNEUROSCI.2270-15.2015 
Matsumura, N., Nishijo, H., Tamura, R., Eifuku, S., Endo, S., \& Ono, T. (1999). Spatial- and task-dependent neuronal responses during real and virtual translocation in the monkey hippocampal formation. The Journal of neuroscience, 19(6), 2381-2393.

https://doi.org/10.1523/JNEUROSCI.19-06-02381.1999

Means, L. W., \& Douglas, R. J. (1970). Effects of hippocampal lesions on cue utilization in spatial discrimination in rats. Journal of comparative and physiological psychology, 73(2), 254-260. https://doi.org/10.1037/h0030205

Milner, B., Corkin, S., \&Teuber, H.L. (1968). Further analysis of the hippocampal amnesic syndrome: 14-year follow-up study of H.M. Neuropsychologia, 6(3), 215-234.

https://doi.org/10.1016/0028-3932(68)90021-3.

Mishkin, M., Ungerleider, L. G, \& Macko, K. A. (1983). Object vision and spatial vision: two cortical pathways. Trends in neurosciences, 1983;6, 414-417 https://doi.org/10.1016/01662236(83)90190-X

Morris RGM (2008). Scholarpedia, 3(8):6315.doi:10.4249/scholarpedia.6315

Molenberghs, P., Sale, M. V., \& Mattingley, J. B. (2012). Is there a critical lesion site for unilateral spatial neglect? A meta-analysis using activation likelihood estimation. Frontiers in human neuroscience, 6, 78. https://doi.org/10.3389/fnhum.2012.00078

Mullally, S. L., \& Maguire, E. A. (2011). A new role for the parahippocampal cortex in representing space. The Journal of neuroscience, 31(20), 7441-7449.

https://doi.org/10.1523/JNEUROSCI.0267-11.2011

Nasr, S., Liu, N., Devaney, K. J., Yue, X., Rajimehr, R., Ungerleider, L. G., \& Tootell, R. B. (2011). Scene-selective cortical regions in human and nonhuman primates. The Journal of 
neuroscience : the official journal of the Society for Neuroscience, 31(39), 13771-13785.

https://doi.org/10.1523/JNEUROSCI.2792-11.2011

Neggers, S. F., Van der Lubbe, R. H., Ramsey, N. F., \& Postma, A. (2006). Interactions

between ego- and allocentric neuronal representations of space. Neurolmage, 31(1), 320-

331. https://doi.org/10.1016/j.neuroimage.2005.12.028

Nitz D. A. (2006). Tracking route progression in the posterior parietal cortex. Neuron, 49(5),

747-756. https://doi.org/10.1016/j.neuron.2006.01.037

O'Keefe, J., Burgess, N., Donnett, J. G., Jeffery, K. J., \& Maguire, E. A. (1998). Place cells, navigational accuracy, and the human hippocampus. Philosophical transactions of the Royal Society of London. Series B, Biological sciences, 353(1373), 1333-1340.

https://doi.org/10.1098/rstb.1998.0287

O'Keefe, J., \& Dostrovsky, J. (1971). The hippocampus as a spatial map. Preliminary evidence from unit activity in the freely-moving rat. Brain research, 34(1), 171-175.

https://doi.org/10.1016/0006-8993(71)90358-1

Packard, M. G., \& McGaugh, J. L. (1992). Double dissociation of fornix and caudate nucleus lesions on acquisition of two water maze tasks: further evidence for multiple memory systems. Behavioral neuroscience, 106(3), 439-446. https://doi.org/10.1037//07357044.106.3.439

Packard, M. G., \& McGaugh, J. L. (1996). Inactivation of hippocampus or caudate nucleus with lidocaine differentially affects expression of place and response learning. Neurobiology of learning and memory, 65(1), 65-72. https://doi.org/10.1006/nlme.1996.0007 
Park, S., Brady, T. F., Greene, M. R., \& Oliva, A. (2011). Disentangling scene content from spatial boundary: complementary roles for the parahippocampal place area and lateral occipital complex in representing real-world scenes. The Journal of neuroscience, 31(4), 1333-1340. https://doi.org/10.1523/JNEUROSCI.3885-10.2011

Park, S., \& Chun, M. M. (2009). Different roles of the parahippocampal place area (PPA) and retrosplenial cortex (RSC) in panoramic scene perception. Neurolmage, 47(4), 1747-1756. https://doi.org/10.1016/j.neuroimage.2009.04.058

Park, S., Konkle, T., \& Oliva, A. (2015). Parametric Coding of the Size and Clutter of Natural Scenes in the Human Brain. Cerebral cortex, 25(7), 1792-1805. https://doi.org/10.1093/cercor/bht418

Patai, E. Z., Javadi, A. H., Ozubko, J. D., O'Callaghan, A., Ji, S., Robin, J., Grady, C., Winocur, G., Rosenbaum, R. S., Moscovitch, M., \& Spiers, H. J. (2019). Hippocampal and Retrosplenial Goal Distance Coding After Long-term Consolidation of a Real-World Environment. Cerebral cortex (New York, N.Y. : 1991), 29(6), 2748-2758. https://doi.org/10.1093/cercor/bhz044 Penny WD, Friston KJ (2007), Scholarpedia, 2(5):1478.

Persichetti, A. S., \& Dilks, D. D. (2016). Perceived egocentric distance sensitivity and invariance across scene-selective cortex. Cortex; a journal devoted to the study of the nervous system and behavior, 77, 155-163. https://doi.org/10.1016/j.cortex.2016.02.006

Persichetti, A. S., \& Dilks, D. D. (2019). Distinct representations of spatial and categorical relationships across human scene-selective cortex. Proceedings of the National Academy of Sciences of the United States of America, 116(42), 21312-21317. https://doi.org/10.1073/pnas.1903057116 
Potegal M. (1969). Role of the caudate nucleus in spatial orientation of rats. Journal of comparative and physiological psychology, 69(4), 756-764.

https://doi.org/10.1037/h0028168

Robertson, R. G., Rolls, E. T., Georges-François, P., \& Panzeri, S. (1999). Head direction cells in the primate pre-subiculum. Hippocampus, 9(3), 206-219.

https://doi.org/10.1002/(SICI)1098-1063(1999)9:3<206::AID-HIPO2>3.0.CO;2-H

Robertson, C. E., Hermann, K. L., Mynick, A., Kravitz, D. J., \& Kanwisher, N. (2016). Neural Representations Integrate the Current Field of View with the Remembered $360^{\circ}$ Panorama in Scene-Selective Cortex. Current biology : CB, 26(18), 2463-2468.

https://doi.org/10.1016/j.cub.2016.07.002

Rosenbaum, R. S., Ziegler, M., Winocur, G., Grady, C. L., \& Moscovitch, M. (2004). "I have often walked down this street before": fMRI studies on the hippocampus and other structures during mental navigation of an old environment. Hippocampus, 14(7), 826-835. https://doi.org/10.1002/hipo.10218

Schindler, A., \& Bartels, A. (2013). Parietal cortex codes for egocentric space beyond the field of view. Current biology : CB, 23(2), 177-182.

https://doi.org/10.1016/j.cub.2012.11.060

Scoville, W. B., \& Milner, B. (1957). Loss of recent memory after bilateral hippocampal lesions. Journal of neurology, neurosurgery, and psychiatry, 20(1), 11-21.

https://doi.org/10.1136/jnnp.20.1.11 
Snyder, L. H., Grieve, K. L., Brotchie, P., \& Andersen, R. A. (1998). Separate body- and worldreferenced representations of visual space in parietal cortex. Nature, 394(6696), 887-891. https://doi.org/10.1038/29777

Spiers, H. J., \& Maguire, E. A. (2007). A navigational guidance system in the human brain. Hippocampus, 17(8), 618-626. https://doi.org/10.1002/hipo.20298

Stein J. F. (1992). The representation of egocentric space in the posterior parietal cortex.

The Behavioral and brain sciences, 15(4), 691-700.

https://doi.org/10.1017/S0140525X00072605

Stein, D. G., \& Kimble, D. P. (1966). Effects of hippocampal lesions and posttrial strychnine administration on maze behavior in the rat. Journal of comparative and physiological psychology, 62(2), 243-249. https://doi.org/10.1037/h0023660.

Suthana, N. A., Donix, M., Wozny, D. R., Bazih, A., Jones, M., Heidemann, R. M., Trampel, R., Ekstrom, A. D., Scharf, M., Knowlton, B., Turner, R., \& Bookheimer, S. Y. (2015). Highresolution 7T fMRI of Human Hippocampal Subfields during Associative Learning. Journal of cognitive neuroscience, 27(6), 1194-1206. https://doi.org/10.1162/jocn_a_00772

Taube J. S. (2007). The head direction signal: origins and sensory-motor integration. Annual review of neuroscience, 30, 181-207.

https://doi.org/10.1146/annurev.neuro.29.051605.112854

Taube JS, Valerio S, Yoder RM (2013). Is navigation in virtual reality with FMRI really navigation? Journal of Cognitive Neuroscience, 25(7):1008-1019. doi: 10.1162/jocn_a_00386. 
Troiani, V., Stigliani, A., Smith, M. E., \& Epstein, R. A. (2014). Multiple object properties drive scene-selective regions. Cerebral cortex, 24(4), 883-897.

https://doi.org/10.1093/cercor/bhs364

Vass, L. K., \& Epstein, R. A. (2013). Abstract representations of location and facing direction in the human brain. The Journal of neuroscience : the official journal of the Society for Neuroscience, 33(14), 6133-6142. https://doi.org/10.1523/JNEUROSCI.3873-12.2013 Vass, L. K., \& Epstein, R. A. (2017). Common Neural Representations for Visually Guided Reorientation and Spatial Imagery. Cerebral cortex (New York, N.Y. : 1991), 27(2), 14571471. https://doi.org/10.1093/cercor/bhv343

Wegman, J., Tyborowska, A., \& Janzen, G. (2014). Encoding and retrieval of landmarkrelated spatial cues during navigation: an fMRI study. Hippocampus, 24(7), 853-868. https://doi.org/10.1002/hipo.22275

Weniger, G., Ruhleder, M., Wolf, S., Lange, C., \& Irle, E. (2009). Egocentric memory impaired and allocentric memory intact as assessed by virtual reality in subjects with unilateral parietal cortex lesions. Neuropsychologia, 47(1), 59-69.

https://doi.org/10.1016/j.neuropsychologia.2008.08.018

Whitlock, J. R. (2017). Posterior parietal cortex Current Biology, 27(14), R691-R695, https://doi.org/10.1016/j.cub.2017.06.007

Wilkinson, L., \& Jahanshahi, M. (2007). The striatum and probabilistic implicit sequence learning. Brain research, 1137(1), 117-130. https://doi.org/10.1016/j.brainres.2006.12.051

Witter M. P. (1993). Organization of the entorhinal-hippocampal system: a review of current anatomical data. Hippocampus, 3, 33-44. https://doi.org/10.1002/hipo.1993.4500030707 
Wolbers, T., Weiller, C., \& Büchel, C. (2004). Neural foundations of emerging route knowledge in complex spatial environments. Brain research. Cognitive brain research, 21(3), 401-411. https://doi.org/10.1016/j.cogbrainres.2004.06.013

Wolbers, T., \& Büchel, C. (2005). Dissociable retrosplenial and hippocampal contributions to successful formation of survey representations. The Journal of neuroscience : the official journal of the Society for Neuroscience, 25(13), 3333-3340.

https://doi.org/10.1523/JNEUROSCI.4705-04.2005

Zhang, H., \& Ekstrom, A. (2013). Human neural systems underlying rigid and flexible forms of allocentric spatial representation. Human brain mapping, 34(5), 1070-1087.

https://doi.org/10.1002/hbm.21494 